\title{
Transcriptome Profiles of Anopheles Gambiae Harboring Natural Low-Level Plasmodium Infection Reveal Adaptive Advantages For The Mosquito
}

\author{
Ann Carr \\ Vanderbilt University \\ David C. Rinker \\ Vanderbilt University \\ Yuemei Dong \\ Johns Hopkins University \\ George Dimopoulos \\ Johns Hopkins University \\ Laurence J. Zwiebel ( $\square$ l.zwiebel@vanderbilt.edu ) \\ Vanderbilt University
}

\section{Research Article}

Keywords: Anopheline, Plasmodium, mosquitoes, transcriptome

Posted Date: September 9th, 2021

DOl: https://doi.org/10.21203/rs.3.rs-881468/v1

License: (c) (i) This work is licensed under a Creative Commons Attribution 4.0 International License.

Read Full License

Version of Record: A version of this preprint was published at Scientific Reports on November 19th, 2021.

See the published version at https://doi.org/10.1038/s41598-021-01842-x. 


\section{Transcriptome Profiles of Anopheles gambiae Harboring Natural Low-Level Plasmodium Infection Reveal Adaptive Advantages for the Mosquito}

Ann Carra , David C. Rinker ${ }^{b}$, Yuemei Dong ${ }^{c}$, George Dimopoulos ${ }^{c}$ and Laurence J. Zwiebel ${ }^{a *}$

a Department of Biological Sciences, Vanderbilt University, Nashville, TN 37235, USA

${ }^{b}$ Department of Chemistry, Vanderbilt University

${ }^{c}$ W. Harry Feinstone Department of Molecular Microbiology and Immunology, Bloomberg School of Public Health, Johns Hopkins University, Baltimore, MD 21205, USA

*Correspondence: I.zwiebel@vanderbilt.edu 


\section{Abstract}

Anopheline mosquitoes are the sole vectors for the Plasmodium pathogens responsible for malaria, which is among the oldest and most devastating of human diseases. The continuing global impact of malaria reflects the evolutionary success of a complex vector-pathogen relationship that accordingly has been the long-term focus of both debate and study. An open question in the biology of malaria transmission is the impact of naturally occurring low-level Plasmodium infections of the vector on the mosquito's health and longevity as well as critical behaviors such as hostpreference/seeking. To begin to answer this, we have completed a comparative RNAseq-based transcriptome profile study examining the effect of biologically salient, salivary gland transmissionstage Plasmodium infection on the molecular physiology of Anopheles gambiae s.s. head, sensory appendage, and salivary glands. When compared with their uninfected counterparts, Plasmodium infected mosquitoes exhibit increased transcript abundance of genes associated with olfactory acuity as well as a range of synergistic processes that align with increased fitness based on both anti-aging and reproductive advantages. Taken together, these data argue against the long-held paradigm that malaria infection is pathogenic for anophelines and, instead, suggests there are biological and evolutionary advantages for the mosquito that drive the preservation of its high vectorial capacity. 


\section{Introduction}

Globally, malaria remains the most endemic infectious/mosquito-borne disease, with over 500 million cases per annum, and putative vectors present in almost 100 countries worldwide placing up to $40 \%$ of the world's population at risk (malERA, 2011). Human malaria is the result of pathogenic infection by five species of unicellular Plasmodium Haemosporidians (WHO, 2020). Of utmost importance is Plasmodium falciparum, which causes severe health complications and the highest human mortality rate (Olliaro, 2008). Plasmodium parasites are solely vectored between humans by mosquitoes within the genus Anopheles (Coetzee and Koekemoer, 2013; Sinka et al., 2012). Anopheles mosquitoes are anautogenous; females require a blood meal for reproduction and replenishment of energy stores (Hagan (2018). Importantly, An. gambiae adult females are only infectious to humans when the Plasmodia they harbor complete a multi-stage sporogonic cycle and successfully invade the mosquito's salivary glands. Despite the complexity of the interlaced Anopheles-Plasmodium life cycles and the inherent ecological and environmental challenges, it is often surprising that in malariaendemic regions of Sub-Saharan Africa, where there is a high disease prevalence, the percentage of infectious mosquitoes within surveys of Anopheles populations is actually very low, typically ranging from $1 \%$ to $10 \%$ (Ogola et al., 2018). Blood feeding, which is carried out solely by adult females, is a central factor that positively affects the complex Anopheles-Plasmodium paradigm. As such, Anopheles blood-meal host-seeking and preference are critical behaviors for regulating the synergistic processes that ultimately impact the mosquito's vectorial capacity. Indeed, several studies support a hypothesis that pathogenic malaria in humans and Plasmodium infection of vectors may modulate mosquito behavior and physiology to increase the likelihood of pathogen transmission 
(Anderson et al., 1999; Lacroix et al., 2005; Stanczyk et al., 2019; Stanczyk et al., 2017). However, many of these and other efforts to examine the relationship between Anopheles and Plasmodium have for experimental simplicity often resorted to employing unnaturally high mosquito infection intensities that might have artifactually altered the natural relationships between Anopheles and Plasmodium (Ferguson and Read, 2004; Pinheiro-Silva et al., 2015; Smallegange et al., 2013; Stanczyk et al., 2017).

To begin, the vast majority of anopheline mosquitoes that ingest Plasmodium gametocyte-infected blood meals never become infectious (Belachew, 2018). In controlled laboratory studies, only 5-10\% of ingested Plasmodium gametocytes successfully differentiate into ookinetes; of those, less than $0.3 \%$ form oocysts, giving rise to approximately 1-2 oocysts per mosquito midgut (Belachew, 2018; Vaughan et al., 1994). This closely mirrors West African field studies indicating that the majority of malaria-infected mosquitoes are within that range and only rarely are trapped mosquitoes observed with more than 5 oocysts per midgut (Collins and Besansky, 1994). In that light, it is reasonable to suggest that when investigating salient biological relationships between Anopheles and Plasmodium in the laboratory, the relative intensity of mosquito infections should align with these natural levels. The high malaria infection intensities that are often employed in studies to provide high infection prevalence are likely to significantly alter the Anopheles-Plasmodium paradigm potentially giving rise to potentially biologically irrelevant shifts in mosquito gene expression, physiology and behavior. We have deliberately employed low gametocytemia and, thereby, biologically relevant $P$. falciparum blood meals to examine how salivary-gland-stage sporozoite infection influences the transcriptome of female An. gambiae head (including sensory appendages) and salivary glands, henceforth referred to 
as 'head'. These data suggest that natural levels of malaria infection align with enhanced olfactory sensitivity that impacts chemosensory driven host preference. Moreover, and surprisingly, natural levels of Plasmodium infection appear to provide a broad and synergistically beneficial modulation of mosquito fitness that underlies and may indeed promote the evolutionary stability of the AnophelesPlasmodium paradigm that continues to drive global malaria transmission.

\section{Results and Discussion}

\section{Experimental Design \& Establishment of Optimal Gametocytemia}

Our study was designed to rigorously compare the impact of biologically salient $P$. falciparum infections on the An. gambiae head (encompassing brain, sensory appendages, and salivary glands) transcriptome. An experimental paradigm incorporating triplicate biological replicates (21 samples per replicate) was designed wherein 5-day-old females were blood fed on either $P$. falciparum-infected (treatment) or naïve (control) human blood meals and thereafter reared for 18 days prior to collection and dissection for downstream molecular analyses. This paradigm allows for completion of the mosquito sporogonic cycle as well as sporozoite invasion of the salivary glands in treated (infected) mosquitoes (Figure 1A), thus ensuring that mosquitoes harboring sporozoites in their salivary glands are examined. Within this study, the importance of establishing and utilizing the low-level intensities representative of natural biological infections was prioritized, in spite of the experimental challenges resulting from significant reductions in prevalence (Collins and Besansky, 1994). To achieve this goal, approximately $14 \%$ of the treatment mosquitoes were sampled 8 days after blood meals, and their midguts were examined for the presence of $P$. falciparum oocysts. Preliminary studies indicated that mosquitoes that had blood fed on human blood supplemented with $P$. falciparum NF54W at a final 
gametocytemia of $0.15 \%, 0.05 \%$, and $0.01 \%$ resulted in median/upper threshold oocyst counts that were significantly higher than the optimal levels of 1-oocyst/midgut median and 3-6 oocysts/midgut upper levels required. Iterative efforts established an optimal gametocyte dilution of $0.008 \%$ to meet those criteria, which was thereafter used for all treatments in this study (Table 1). The heads (including sensory appendages and salivary glands) were used for preparation of total RNA (for sequencing) and salivary tissues for genomic DNA (gDNA) for PCR validation of the presence of $P$. falciparum sporozoite-treated individual mosquitoes. These PCR-based analyses utilized primers designed to detect the $P$. falciparum circumsporozoite (CS) gene as well as the An. gambiae ribosomal protein s7 (AgRsp7) gene as an internal standard (Coppi et al., 2011). This analysis revealed that, of the 176 treatment mosquitoes that took infected blood meals and survived to 23 days of age, 65 were confirmed as having salivary-gland-stage sporozoites, representing an overall prevalence of $\sim 37 \%$ (Figure 2). Consistent survivor rates of $\sim 50 \%$ were observed between treatment and control An. gambiae lines. The observed survival rate parallels previous studies documenting 20day-old An. gambiae laboratory survival rates (Huestis et al., 2017).

\section{RNAseq/Bioinformatics}

Transcriptome profiling was conducted using total RNA extracted from triplicate An. gambiae treatment (infected; T1-3) and control (uninfected; C1-3) heads. Following sequence trimming and quality control bioinformatic pipelines, individual data sets were aligned to the An. gambiae PEST strain genome. Sequencing of the T1, T2, and T3 P. falciparum-infected (treatment) An. gambiae head libraries generated a total of over 254 million reads mapping to $83 \%, 83 \%$, and $85 \%$ respectively of the An. gambiae genome (VectorBase-53_AgambiaePEST; Table 2). Likewise, 
Illumina sequencing of the C1, C2, and C3 control (uninfected) An. gambiae head libraries generated a total of 426 million reads mapping to $81 \%, 80 \%$, and $81 \%$ respectively of the An. gambiae genome (VectorBase-53_AgambiaePEST; Table 2). Uniquely mapped reads were counted using the program HTSeq. DESeq2 statistical analysis identified 5,765 annotated transcripts present in significantly different abundances between treatment and control replicates $(p<0.05$; Figure $3 \mathrm{~A})$. These annotated transcripts represent a transcriptional shift of $36 \%$ of the An. gambiae head transcriptome. Of these, 2,776 transcripts were present in significantly higher abundances in treatment replicates and the remaining 2,989 transcripts present in significantly higher abundance in control replicates $(p<$ 0.05). With such a large transcriptional shift observed between treatment and control An. gambiae heads, both principal component analysis (PCA) and Pearson's correlation coefficient (PCC) calculations were performed to verify validity. PCC calculations for the three treatment and three control An. gambiae head libraries returned $r$ values of 0.9429 and 0.9770 , respectively, indicating a strong association among biological replicates (Table 2). Additionally, PCA determined that $83 \%$ of observed variance between the six library datasets was attributed to the presence/absence of $P$. falciparum infection, with negligible variance attributed to biological replication (Figure 3B).

GO annotation using OmicsBox assigned 4,976 GO-terms (Level II) to 2,832 transcripts of the 5,765 differentially abundant transcripts (control and treatment libraries). Of the total annotated transcripts, 1,291 were elevated in treatment libraries and 1,541 transcripts elevated in control libraries, with approximately $10 \%$ of the transcripts having no known function or characterization (Figure 4). Transcripts with no clear molecular function via GO were further BLASTed using Blast2GO and the GeneBank non-redundant protein database to assist in further functional analysis. The resultant 
assigned GO annotations are representative of a diverse array of biological processes and molecular functions that make interpretation difficult. Taken together, these analyses reveal a broad transcriptome profile shift that spans a wide range of cellular processes, some of which are likely to impact the behavior, physiology and ultimately the vectorial capacity of An. gambiae carrying biologically relevant $P$. falciparum sporozoite infections.

\section{Plasmodium Manipulation of Mosquito Chemosensory Mechanisms}

To examine the potential for behavioral modification of infected mosquitoes, our initial focus was to examine transcriptome modulations directly relevant to the chemosensory systems of An. gambiae.

These systems collectively play an essential role in establishing and maintaining critically important behaviors, including the blood-meal host preference of female mosquitoes (Montell and Zwiebel, 2016; Takken and Verhulst, 2013).

Odorant Receptors: The transcript abundance of several An. gambiae odorant receptors (AgORs) were highly differentiated between $P$. falciparum sporozoite-infected (sporozoite; treatment) and uninfected (control) heads. Indeed, 60 of the $71 \mathrm{AgORs}$ and the OR co-receptor (Orco) that were detected in sequencing libraries were consistently more abundant in heads of infected mosquitoes, with 19 displaying statistically significant induction (Figure 5A). Transcripts encoding the remaining 11 AgORs (AgOR1, AgOR3, AgOR4, AgOR26, AgOR36, AgOR45, AgOR46, AgOR52, AgOR60, AgOR62 and AgOR72) showed an insignificant trend of less abundance in samples from infected mosquitoes (Figure 5A). With the near complete representation of AgORs (95\% of annotated An. gambiae ORs), it is evident that sporozoite infection significantly impacts the levels of these ORs within mosquito chemosensory structures. The majority of anopheline ORs are functionally 
characterized, and we have previously integrated OR functional RNAseq data to model the receptivity profile for the antennae of An. gambiae following their initial bloodmeal (Rinker et al., 2013a). Transcriptome profiling studies together with behavioral analyses support the hypothesis that the sensitivity of the anopheline olfactory system is directly associated with the relative abundance of olfactory transcripts (Rinker et al., 2013a; Rinker et al., 2013b; Wolff and Riffell, 2018). Applying this approach here suggests that the large number of significantly abundant AgOR transcripts associated with infected An. gambiae indicates substantial increases in odorant receptivity (Figure 6). In fact, AgOR receptivity modelling identified 70 odorants, many of which are components of human sweat, with significantly increased receptivity in Plasmodium-infected mosquitoes than in uninfected controls. Of these, several [including L-lactic-acid, 1-octen-3-ol and 4-methylphenol] are human sweat components that have been shown to be attractive kairomones for host-seeking female anophelines (Cork and Park, 1996; Smallegange et al., 2005). Notably, lactic acid showed a $217 \%$ increase in AgOR receptivity in treatments. Furthermore, the significant increases in receptivity to 1-butanol (247\%), 3-methyl-1-butanol (260\%), and 2,3-butanedione (252\%) align with laboratory olfactometry and semi-field studies, indicating that these compounds significantly increase the number of anopheline catches when added to MMX traps baited with $\mathrm{CO}_{2}$ and a basic blend of amine/carboxylic acid attractants (Verhulst et al., 2011).

These putative increases of olfactory acuity in Plasmodium-infected anopheline mosquitoes are consistent with behavioral bioassay and surveillance studies that determined Plasmodium infections to increase mosquito bite frequency while maintaining host-preference plasticity (Cator et al., 2014; Vantaux et al., 2015). That said, our receptivity modelling does not align with recent studies that examined peripheral olfactory responses in P. falciparum-infected An. gambiae (Stanczyk et al., 
2019). It is likely that the significantly higher infection intensities used in that study over-challenged An. gambiae infection-responsive systems, such as the innate immune system, resulting in unintended fitness consequences that altered host-seeking and blood-feeding behaviors (Ohm et al., 2016). Because our studies avoid impacts arising from non-natural infection intensities, they can more confidently reveal a clear effect of biologically relevant malaria infections on the anopheline olfactory system. The effect of increasing host seeking and host feeding synergistically support both parasite and mosquito survivorship as well as maximizing vectorial capacity. Interestingly, the presence of polycistronic OR genes in Anopheles (Karner et al., 2015) raises the hypothesis that Plasmodium parasites may have evolved to specifically activate the transcription factors regulating polycistronic ORs to efficiently induce large-scale olfactory modulation. Indeed, all of the AgORs identified in polycistronic cluster 1B (Karner et al., 2015) are present in our sequencing libraries, and one of these AgORs17 was significantly over-represented in malarial mosquitoes; the increased abundance of the remaining five AgORs $(13,15,16,47,55)$ are just below statistical significance (Figure 5A).

Odorant Binding Proteins/Chemosensory proteins: Odorant binding proteins (OBPs), chemosensory proteins (CSPs) and sensory appendage proteins (SAPs) have been postulated to mediate the solubility and transport of odorant molecules within the sensilla lymph (Leal, 2013). A total of $57 \mathrm{AgOBPs} / \mathrm{AgCSPs} / \mathrm{AgSAPs}$ were identified in sequencing datasets, with 47 AgOBPs/AgCSPs more abundant in heads of infected mosquitoes (17 significantly) while two AgOBPs (9 and 25) and a single AgCSP5 were significantly under-represented relative to uninfected controls (Figure 5B). These findings are consistent with microarray-based transcriptome studies in Ae. aegypti that determined dengue viral infection to increase abundance of a subset of OBPs that 
was correlated to increased host feeding and vectorial capacity (Sim et al., 2012). It is reasonable to postulate that Plasmodium infection similarly impacts anopheline mosquitoes to potentially increase host feeding, synergistically supporting parasite and mosquito survivorship as well as maximizing vectorial capacity. AgCSP5 (-0.72-fold; $p$-adj = 0.014; AGAP029127), AgOBP9 (-0.54-fold; $p$-adj = 0.030; AGAP000278), and AgOBP25 (-0.40-fold; p-adj = 0.018; AGAP012320) are the only significantly under-represented (-1.77-fold; ACOM030596) OBP/CSP transcripts (Figure 5B). In any case, the precise role of AcCSP5, AgOBP9, and AgOBP25 along with those of other OBP/CSP genes in An. gambiae olfaction remains enigmatic.

Gustatory Receptors: Phylogenetic-based annotation (Kent et al. 2008; Sparks et al. 2013) along with appendage-specific transcriptome profiling of An. coluzzii, An. gambiae, and Ae. aegypti gustatory receptors (GRs) correlate several orthologous GR genes with the gustatory responses of putative gustatory receptor neurons (GRNs) on the proboscis and labellum. That said, there is a paucity of direct functional data for the majority of An. gambiae gustatory receptors (AgGRs) (Matthews et al., 2016; Pitts et al., 2011; Rinker et al., 2013a). In contrast, AgGR22 (AGAP00999), AgGR23 (AGAP003098), and AgGR24 (AGAP001915), which make up a complex that is required for volatile carbon dioxide $\left(\mathrm{CO}_{2}\right)$ detection critical for blood-feeding behaviors, have been well characterized (Liu et al., 2020). The differential transcriptome profiles for AgGR22 (0.28-fold), AgGR23 (0.31-fold), and AgGR24 (0.34-fold) are only slightly more abundant in sporozoite-infected head transcriptomes than in controls (Figure 7A, red bar) and, not surprisingly, these changes fail to meet the rigorous significance thresholds employed here. This suggests that Plasmodium infection does not significantly impact $\mathrm{CO}_{2}$ sensitivity and is consistent with previous electrophysiology-based 
studies that showed $P$. berghei infections had no effect on An. stephensi maxillary palp neuron sensitivity to $\mathrm{CO}_{2}$ (Grant et al., 2013). Taken together, it appears likely that $P$. falciparum parasites do not manipulate early, long-range anopheline host-seeking behaviors, such as $\mathrm{CO}_{2}$ detection (Nguyen et al., 2017).

Beyond the $\mathrm{CO}_{2}$ receptors, we observed a considerable shift in the AgGR transcriptome profile between heads of uninfected and those of sporozoite-infected mosquitoes. A total of $44 \mathrm{AgGRs}$ were differentially detected; transcripts for 40 AgGRs showed a higher abundance and the remaining 4 were less abundant in heads of sporozoite-infected mosquitoes than in controls (Figure 7A). Of these differentially abundant AgGRs, 13 were present at significantly higher levels and none at significantly lower levels in treatments. While the most significantly abundant, AgGR11 (3.43-fold), and seven other AgGRs that are significantly more abundant in treatments remain unannotated, several AgGRs with homology to functionally characterized sugar-responding GRs from Drosophila display significantly higher transcript abundances than controls, suggesting a higher sensitivity to sugary substrates in treatments. These include the DmGR5a orthologs AgGR14 (1.94-fold; $p$-adj $=<0.001$; AGAP006399) and AgGR21 (0.88-fold; p-adj = 0.05; AGAP003260); the DmGR43a ortholog AgGR25 (0.83-fold; $p$-adj $=0.05 ;$ AGAP004727); and the DmGR68a ortholog AgGR9 (2.90-fold; $p$-adj = $<0.0001$; AGAP009805; Figure 7A) (Kent et al., 2008). It is reasonable to hypothesize that because glucose is the primary energy source for both mosquitoes and the Plasmodium sporozoites, it would be advantageous to manipulate its acquisition, uptake and transport, thereby synergistically supporting sporozoite and mosquito health, survival and by extension, malaria transmission (Gary and Foster, 2001; Pumpuni and Beier, 1995). This differential increase in putative sugar-responsive 
AgGRs as a consequence of salivary gland $P$. falciparum infections also correlated with a significant increase in the abundance of several sugar transporters (Supplementary Information). While this is the first identification of a $P$. falciparum-mediated increase in abundance of non- $\mathrm{CO}_{2}$ responsive AgGRs, our data are consistent with previous studies highlighting the overabundance of sugar transporters in the salivary glands of Plasmodium-infected An. gambiae and their importance for sporozoite survival (Pinheiro-Silva et al., 2015). The remaining transcript, AgGR47 (2.60-fold; $p$-adj = 0.05; AGAP005514), found significantly abundant in treatments is a DmGR66a ortholog and putatively functions in bitter/caffeine detection (Kent et al., 2008). It is reasonable to speculate that Plasmodium sporozoites in Anopheles mosquitoes upregulate the deterrent effects of such AgGR neuronal responses to prevent ingestion of detrimental bitter nutrient sources that have been shown to temporally inhibit mosquito sugar and water neurons and reduce nectar feeding of $A n$. quadrimaculatus (Sparks and Dickens, 2016).

Shifting mosquito gustatory profiles to increase sensitivity to bitter and behaviorally aversive semiochemicals therefore increases the likelihood of blood-meal (and or nectar) feeding and consequently pathogen transmission. It is likely that this effect contributes to increased sensitivity and the marked decrease in mortality rate of An. gambiae harboring $P$. falciparum sporozoites when exposed to DEET (N, N-diéthyl-3-méthylbenzamide), a widely used repellent, in comparison with uninfected An. gambiae and other pyrethroid test substrates (Mulatier et al., 2018; Swale et al., 2014). Lastly, the increased sensitivity of AgGR47 observed in sporozoite-infected An. gambiae relative to naïve controls may reflect age-related nutrient deficiencies in controls. In aging Drosophila, 
nutrient deprivation dramatically alters feeding behaviors, including the depotentiation of responses to bitter (Maruzs et al., 2019).

Ionotropic Receptors: Ionotropic receptors are an ancient family of chemosensory receptors evolutionarily derived from ionotropic glutamate receptors (Croset et al., 2010) that are associated with olfactory responses to amines and carboxylic acids that have been identified as important semiochemicals closely associated with host-preference and seeking (AgIRs; Montell and Zwiebel, 2016). As is true in other insects, AgIR8a, AgR25a and AgIR76b likely function as co-receptors along with odorant-recognizing AgIRs that 'tune' responses to host cues (Liu et al., 2010; Pitts et al., 2017). In An. gambiae, AgIR25a together with AgIR76b and AgIR8a function in the detection of amines and carboxylic acids, respectively (Pitts et al., 2017) both of these chemical classes have been directly implicated in anopheline host seeking (Smallegange et al., 2005; Takken and Verhulst, 2013). Interestingly, the abundance of AgIR76b transcripts was significantly enhanced (0.55-fold; $p$-adj $=$ 0.05; AGAP011968) in infected mosquito heads while the levels of AgIR25a and AgIR8a were not significantly different (Figure 7B). Transcripts for 38 of the 49 odorant-tuning AgIRs detected here were found to be more abundant in sporozoite-infected head transcriptomes; only 11 were downregulated compared with uninfected controls (Figure 7B). Of those upregulated, 18 were significantly more abundant but none were significantly less abundant in treatment groups than controls. Inasmuch as many AgIRs are uncharacterized, including those with the most pronounced and significant differential abundance (AgIR149, AgIR168, and AgIR7203), the implications of these differentially up-/downregulated IRs are difficult to assess. Nevertheless, functional information for a small subset of AgIRs is available and others can be derived from analyses of clear orthologs in $A e$. 
aegypti, An. gambiae, An. sinensis and Drosophila melanogaster. This allows us to identify several AgIRs significantly more abundant in treatment groups (Figure 7B) to be associated with both gustation [AgIR7x (3.11-fold; $p$-adj = 0.03; AGAP013520), AgIR93a (0.52-fold; $p$-adj = 0.05; AGAP000256) (Hill et al., 2019), AgIR60a (2.48-fold; p-adj = 0.05; AGAP011943) (Menuz et al., 2014)] and host-seeking behaviors [AgIR75g (1.96-fold; p-adj = 0.003; AGAP013085) (Hill et al., 2019)]. In addition, we observed three receptors localized to the antenna [AgIR31a (1.43-fold; $p$-adj = 0.03; AGAP009014), AgIR68a (2.00-fold; p-adj = 0.003; AGAP007951), and AgIR41t1 (1.44-fold; p$\operatorname{adj}=0.03 ;$ AGAP004432)(Wang et al., 2018)]. While persistence of host seeking and blood feeding might well be advantageous for Plasmodium, it would only be beneficial for their anopheline hosts if these behavioral shifts promoted the acquisition of a successful blood meal.

Transient Receptor Potential Channels: As is true for all insects, An. gambiae transient receptor potential (AgTRP) family members are associated with a variety of sensory modalities such as chemosensation, gustation, thermosensation, hygrosensation, mechanosensation, vision, and intracellular signaling (Montell and Zwiebel, 2016). A total of nine AgTRPs were identified in sequencing libraries, five of which are more abundant (three significantly) in heads of sporozoiteinfected mosquitoes while four AgTRPs (three significantly) are under-represented relative to uninfected controls (Figure 7C). Of these, AgTRPC (canonical; 0.50-fold; $p$-adj $=0.0002$; AGAP000349), AgTRPC (canonical; 0.67-fold; $p$-adj = 0.0004; AGAP008435), and AgTRPM (melastatin; 0.75-fold; $p$-adj $=0.01 ;$ AGAP006825) are all active in thermosensation and feeding. TRPC has been identified as having several physiological roles in insects, including signal transduction, phototransduction, and gustatory sensitivity to $\mathrm{CO}_{2}$ (Badsha et al., 2012). TRPM mediates Drosophila nociception behaviors and putatively critical behaviors for An. gambiae viability 
and survival (Fowler and Montell, 2013). Given the critical roles that AgTRPC and AgTRPM are likely to play in mosquito host seeking and blood feeding, manipulation of these transcripts would likely be advantageous for Plasmodium to ensure maximum host contact rate and transmission. TRP-Painless and TRP-Pyrexia function in thermosensation and have been documented to mediate avoidance of noxious high temperatures in $D$. melanogaster (Sokabe and Tominaga, 2009). It is possible the significantly reduced abundance of AgTRP-Painless (-0.78-fold; $p$-adj $=0.01$; AGAP001243) and AgTRP-Pyrexia (-0.75-fold; $p$-adj = 0.0003; AGAP010269) in treatment groups compared with naïve controls is related to a loss of nociception.

The remaining An. gambiae TRP identified as significantly differentially abundant between treatments and controls functions as a mechanoreceptor with roles in Drosophila hygrosensation. AgTRPWaterwitch which is significantly less abundant in treatment tissues $(-0.70$-fold; $p$-adj $=0.001$; AGAP000361) is homologous to the DmTRP-Waterwitch that functions in dry-air detection (Bohbot et al., 2014). Moisture detection, or importantly the lack thereof, mediates flight and host-seeking behavior in anopheline mosquitoes such that augmenting mosquito traps with moist air significantly increases flight activity and attraction of wild Aedes, Anopheles and Culex mosquitoes (Cribellier et al., 2020). It is likely that upregulation of chemo/thermosensory TRPs as well as the inverse regulation of $\mathrm{Ag}$-Waterwitch optimizes persistent host seeking and blood feeding and is consistent with studies showing that Anopheles mosquitoes harboring Plasmodium sporozoites exhibit more persistent host-seeking behavior than uninfected controls (Ferguson and Read, 2004).

Pickpocket Channel Proteins/Sensory Neuron Membrane Proteins: We identified 18 An. gambiae pickpocket channel proteins (AgPPKs) of which transcripts were predominately more abundant in 
heads of sporozoite-infected mosquitoes (Figure 7D). Two of these AgPPKs, AgPPK13-like (0.97-fold; p-adj $=0.004 ;$ AGAP007945) and AgPPK31-like (1.47-fold; p-adj = 0.05; AGAP000657), showed significant enrichment. In D. melanogaster, PPKs have been implicated to function in salt and water taste, although the behavioral implications of these characterized PPKs is unclear (Freeman and Dahanukar, 2015). Similarly, while aedine PPKs are hypothesized to function in a variety of sensory modalities, such as chemosensation, hygrosensation and mechanosensation (Matthews et al., 2016), anopheline PPKs remain largely uncharacterized and the implications of these differentially abundant PPKs remain unclear. Sensory neuron membrane proteins (SNMPs) are both colocalized with ORs and broadly expressed throughout $D$. melanogaster and are postulated to have both chemosensory and non-chemosensory functions (Vogt et al., 2021) with particular roles in dipteran and lepidopteran pheromone detection (Cassau and Krieger, 2020). Both AgSNMP1 and AgSNMP were identified in sequencing libraries, although without significance (Figure 7E). These and other anopheline SNMPs remain uncharacterized, and the implications of differentially abundant AgSNMPs are therefore unclear.

General Neuronal Function: Insect neurotransmitters comprise acetylcholine (ACh), gammaaminobutyric acid (GABA), glutamate (Glu), and select biogenic amines such as dopamine, histamine, octopamine, serotonin and tyramine as well as their respective membrane receptors (Osborne, 1996). In total, 11 subunits for nicotinic ACh receptors (nAChr) and muscarinic ACh receptor subunits (mAChr) were identified with $5 \mathrm{Ag}$-nAchr and $1 \mathrm{Ag}$-mAchr significantly overabundant in the heads of sporozoite-infected An. gambiae: Ag-nAChr2 (0.75-fold; $p$-adj $=0.001$;

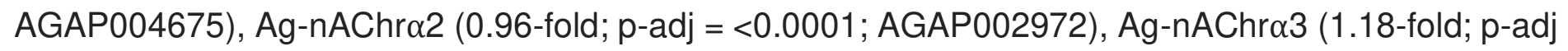
$=<0.0001 ;$ AGAP00329), Ag-nAChra5 (1.25-fold; p-adj = <0.0001; AGAP008588), Ag-nAChr 66 
(1.731-fold; $p$-adj $=<0.0001 ;$ AGAP002152), and Ag-nAChr $\beta 1$ (0.90-fold; $p$-adj $=<0.0001$;

AGAP000966; Figure 8A). Only Ag-nAChra9 (-1.23-fold; $p$-adj $=<0.0001$; AGAP009493) was significantly diminished in heads of sporozoite-infected mosquitoes compared with naïve mosquitoes (Figure $8 \mathrm{~A}$ ). While insect and especially mosquito AChRs (nicotinic and muscarinic) are not well characterized, it has been reported that with age, AChR responses become significantly diminished as a function of oxidative damage and neurodegeneration (Araujo et al., 1990). In Drosophila this is associated with aged-related immune system dysfunction (Nainu et al., 2019). As such, the increased abundance of AgAChrs in sporozoite-infected An. gambiae suggests that malaria infected anopheline mosquitoes are resistant to both aged-related immune system dysfunction and neurodegeneration. A similar logic could apply to the significant overabundance of acetylcholinesterase (Ace; AgAce, 1.18fold; $p$-adj $=<0.0001 ;$ AGAP000466) identified in treated An. gambiae relative to naïve controls (Figure 9A). As such, the increased levels of AChRs in treatment groups could also represent altered baseline levels within mosquitoes that as a result are not yet susceptible to the effects of aging and senescence.

Transcripts for both ionotropic (GABA-A) receptors and metabotropic (GABA-B) receptors were significantly more abundant in treatment groups when compared with naïve controls: AgGABA-A (0.80-fold; p-adj = 0.0007; AGAP006028), AgGABA-B (0.38-fold; p-adj = 0.02; AGAP000038), and AgGABA-B2 (1.14-fold; $p$-adj = <0.0001; AGAP004595; Figure 8B). Transcripts for the GABA processing enzyme glutamate decarboxylase (Gad) displayed a dissimilar differentiation as the abundance of transcripts encoding AgGad1 (-2.92-fold; p-adj =<0.0001; AGAP005866) and AcGad2 $(-1.10$-fold; $p$-adj $=0.0001 ;$ AGAP008904 $)$ were significantly diminished in treatment groups 
compared with controls (Figure 9A). In Drosophila, GABA mechanisms help stabilize neuronal function and maintain tissue homeostasis (Maruzs et al., 2019). As such, the decreased abundance of AgGABAs and increased abundance of AgGads in naïve controls may actually reduce agingrelated neurodegeneration to help maintain neuronal homeostasis.

Similarly, transcripts for NMDA and non-NMDA types of anopheline glutamate receptors were identified in sequencing libraries and predominately under-represented in heads of infected $A n$. gambiae. Of the eight glutamate receptor transcripts identified, none was significantly abundant in heads of sporozoite-infected mosquitoes; in contrast, six NMDA and non-NMDA glutamate receptors-AgGluRIla (-1.27-fold; $p$-adj $=<0.0001 ;$ AGAP000803), AgGluRIld (-0.54-fold; $p$-adj $=$ 0.003; AGAP002797) AgGluRlle (-0.81-fold; $p$-adj = <0.0001; AGAP012447), AgNMDAR1 (-1.23-fold; p-adj $=<0.0001 ;$ AGAP001478), AgNMDAR2 (-0.32-fold; $p$-adj = 0.005; AGAP012429) and AgNMDAR3 (-0.98-fold; $p$-adj = 0.002; AGAP005527)—were significantly diminished in sporozoiteinfected mosquitoes compared with naïve controls (Figure $8 \mathrm{C}$ ). It is possible that in light of the ability of these receptors to function as immunomodulators of inflammatory responses (Hodo et al., 2020), the overabundance of NMDA and non-NMDA glutamate receptors in naïve mosquitoes reflects either an aging-related immune system dysfunction or neuronal pathology. Transcripts encoding the receptors for several peptide biogenic amines, including dopamine, histamine, octopamine, serotonin and tyramine, which function as anopheline neuromodulators and neurotransmitters were identified in our libraries. With the exception of two pairs of histamine and octopamine receptor transcripts that were similarly up-/downregulated, most biogenic amine receptors showed no significant differential abundance in heads of sporozoite-infected An. gambiae relative to naïve controls (Figure 8D-G). 
Transcripts encoding several biogenic amine processors and transporters were identified in similarly up-/downregulated pairs with no clear distinction between treatment and naïve controls (Figure 9A, 9D). Insect neuropeptides are essential neuromodulators that regulate a variety of physiological and behavioral functions. Several neuropeptide transcripts identified in sequencing libraries were shown to be significantly overabundant in the heads of sporozoite-infected An. gambiae (Figure 9B). Interestingly, there were also four neuropeptide transcripts and their correlating receptors that were significantly diminished in treatment heads compared with naïve controls: allatostatin 1 (AgAs1; -0.50fold; $p$-adj = 0.001; AGAP003712), allatostatin 2 (AgAs2; -0.41-fold; $p$-adj = 0.01; AGAP010157), FMRFamide (AgFMRF, -1.34-fold; $p$-adj $=<0.0001$; AGAP005518) and sickie (AgSickie; -1.66-fold; $p$ $\operatorname{adj}=<0.0001 ;$ AGAP009424) were directly or indirectly correlated with insect stress and immune response (Figure 9B-C). In Ae. Aegypti, allatostatin interacts with hemocytes to increase humoral immune responses to combat cellular stress and inflammation (Hernandez-Martinez et al., 2017). FMRFamide signaling in Drosophila increases in response to cellular stress and inflammation to promote stress-induced sleep and cellular recovery to help bolster immune function (Lenz et al., 2015). Sickie acts directly on Drosophila innate immunity by activating the transcription factor Relish and subsequent transcription of immune defense antimicrobial peptides (AMPs; Govind, 2004 \#31754). Neuropeptide F (NPF) also acts indirectly to modulate stress responses through activation of downstream signaling pathways. In dipterans, NPF independently activates insulin signaling pathways and coordinately the target or rapamycin (TOR) pathway (Dhara et al., 2013). TOR pathway is an insect nutrient-sensing pathway that regulates cellular metabolism and homeostasis in response to nutrient flux and stress (Lee et al., 2008; Wullschleger et al., 2006). TOR signaling naturally increases with age and increasing cellular dysfunction and stress (Katewa and Kapahi, 
2010). With a substantial number of neuropeptides/hormones and their correlating receptors significantly abundant in heads of sporozoite-infected An. gambiae and functioning to maintain healthy mosquito cells and tissues, the decreased abundance of these select five neuropeptides in infected mosquitoes is puzzling. If the "normal' transcriptome profile of naïve mosquitoes reflect changes associated with aging-related immune system dysfunction and the loss of cellular homeostasis, or some other age-related pathology, then one could hypothesize that infected mosquitoes might be refractory to those effects.

Immune System: Activation of the immune system in response to infection is energetically costly and likely results in decreased fitness reflected by other life history traits with the potential to impact vectorial capacity. Mosquito anti-Plasmodium immunity is stage specific and involves diverse defense processes, although the extent of immune defense in salivary-gland-stage infected mosquitoes, and especially the head and salivary glands (post sporozoite salivary gland invasion), remains unclear (Belachew, 2018). Since Plasmodium, unlike arboviruses, does not establish infection in the mosquito head, the immunity-related transcriptome changes observed in our study are likely to mostly derive from the combined head (including sensory appendages) and salivary gland transcriptomes. In heads of sporozoite-infected and control sequencing libraries, several components of An. gambiae humoral and cellular, innate immune defenses were identified. An. gambiae Pattern Recognition Receptors (PRRs) include 6 fibrinogen proteins (FBGs), 7 3-glucan binding proteins (3GNBPs), 7 peptidoglycan recognition proteins (PGRPs), 24 leucine-rich repeat immune proteins (LRIMs) including Plasmodium infection responsive leucine-rich proteins, 13 thioester-containing proteins (TEPs), and 2 members of C-type lectin family proteins (CTLs)—AgCTL4 and AgCTLM2 (Figure 10A, 10B). A large portion of the PRR transcripts were not present at significantly differential abundances between heads of 
sporozoite-infected and control mosquitoes; indeed, neither of the transcripts encoding AgCTLMA2 or AgCTL4 displayed significant differential abundance.

Anopheline TEPs are antimicrobial and antiparasitic PRRs, complement-like factors that drive pathogen clearance through lysis and melanization (Blandin et al., 2004). Of those, only AgTEP13 (0.34-fold; p-adj = 0.03; AGAP008407) was significantly more abundant in heads of sporozoiteinfected mosquitoes, and AgTEP1 (-1.95; $\mathrm{p}$-adj $=<0.0001 ;$ AGAP010815) and AgTEP12 (-0.94; $\mathrm{p}$-adj $=0.011$; AGAP008654) significantly diminished in treatment groups compared with controls (Figure 10A). While the roles of TEP12 and TEP13 in anopheline immune responses are not currently known, the well-studied TEP1 functions as an enhancer of mosquito phagocytosis (Kumar et al., 2018). Since TEP1 is incapable of colocalizing and killing Plasmodium sporozoites, its decreased abundance in treatment groups is not unexpected (Blandin et al., 2004) (Yan and Hillyer, 2019). As such, the increased abundance of AgTEP1 in naïve controls likely reflects aging-related immune system dysfunction, as aging decreases phagocytic efficiency and contributes to immunosenescence (Maruzs et al., 2019). LRIMs and APLs are PPRs that mediate innate immune surveillance and response. Transcripts for 20 LRIMs and APLs were downregulated in heads of infected mosquitoes relative to naïve controls, while 4 of the 24 An. gambiae LRIMs were more abundant in treatments. Of the 4 AgLRIMs upregulated, 2 were significantly more abundant, whereas 9 of the 20 LRIMs and APLs were significantly less abundant in treatments than controls (Figure 10B). These data are largely consistent with previous studies on the transcriptional regulation of LRIMs and APLs in response to Plasmodium, gram-negative/gram-positive bacteria, and fungal challenge (Aguilar et al., 2005; Waterhouse et al., 2010). While AgLRIM12 (0.72-fold) and AgLRIM5 (0.82-fold; ACOM043147) were both significantly more abundant in heads of infected mosquitoes they are not yet conclusively 
associated with Plasmodium infection (Dong et al., 2006a; Waterhouse et al., 2010); it is therefore possible the observed differential abundance of AgLRIMs reflect non-immunity responses or potential aging effects that are only partially impacted by $P$. falciparum infection.

Similarly identified AgFBGs, which function in phagocytosis and complement activation, were predominately diminished in treatments relative to naïve controls, with AgFBG (-1.47-fold; $p$-adj = 0.015; AGAP011231), AgFBG (-1.98-fold; $p$-adj = 0.004; AGAP010869), AgFBG (-1.29-fold; $p$-adj = 0.02; AGAP004917) and AgFBG (-1.29-fold; $p$-adj = 0.007; AGAP006914) significantly reduced (Figure 10B; Kumar et al., 2018). In Drosophila, aging is associated with increased microbial loads and dysbiosis that trigger increased expression of immune genes and stronger immune activation (Garschall and Flatt, 2018). Excessive immune activation ultimately results in immune dysfunction and decreased lifespan (Guo et al., 2014). In that context, the large number of AgLRIMs, AgAPLs and AgFBGs that are more abundant in naïve control transcriptomes may reflect aging-related dysfunction of the An. gambiae immune system. Accelerated immune system dysfunction in naïve controls also rationalizes the observed AgPGRP transcript abundances identified in replicate sequencing libraries. Our data indicate that transcripts for two AgPGRPs that have been characterized as negative regulators of immune IMD signaling defense (Ramirez et al., 2020)—AgPGRP-LB (1.46-fold; $p$-adj = 0.02; AGAP001212) and AgPGRP-LD (0.69-fold; p-adj = 0.05; AGAP005552: Figure 10A)—are significantly more abundant in heads of sporozoite-infected mosquitoes, suggesting a suppression of the IMD immune pathway. The IMD signaling initiation receptor AgPGRP-LC (-0.25-fold; $p$-adj $=0.02$; AGAP005203), identified as significantly reduced in treatments compared with controls, further supports the presence of diminished IMD signaling in sporozoite-infected An. gambiae. PGRP immune regulator deficiency results in unregulated IMD signaling causing dysplasia, inflammation, 
apoptosis, and decreased lifespan (Gupta et al., 2009). Preservation of immune homeostasis would be advantageous for both Anopheles hosts and Plasmodium pathogens to maintain vectorial capacity. It is possible that An. gambiae challenged with biologically relevant, low-level $P$. falciparum results in a mild and prolonged engagement of the mosquito immune system that ultimately primes the system for protection from secondary infection and importantly, the negative impact of agingrelated immune system degeneration (Cooper and Eleftherianos, 2017). Indeed, Plasmodiuminduced anopheline immunological priming may represent an advantageous selective adaptive pressure that maximizes mosquito host fitness and, consequently, vectorial capacity.

Serine proteases constitute the majority of mosquito immune system moderators that are activated in response to pathogen recognition (Kumar et al., 2018). While there are hundreds of serine proteases functioning in a variety of mosquito molecular processes, eight CLIP-domain serine proteases (CLIPs)—SPCLIP1, CLIPA2, CLIPA7, CLIPA8, CLIPA14, CLIPA28, CLIPB14, and CLIPB15mediate P. berghei melanization (Nakhleh et al., 2017; Kumar et al., 2018; Sousa et al., 2020). No AgCLIP transcripts were significantly more abundant in heads of sporozoite-infected mosquitoes, although eight were less abundant in treatment groups for which mRNAs of AgCLIPA7 (-1.34-fold: p$\operatorname{adj}=<0.0001 ;$ AGAP011792), AgCLIPA14 (-1.2-fold; $p$-adj $=<0.0001 ;$ AGAP011788) and AgCLIPA28 (-0.92-fold: $\mathrm{p}$-adj = 0.02; AGAP010968) were significantly under-represented relative to uninfected controls (Figure 10C). These data were expected because anopheline CLIPs predominately moderate melanization of rodent Plasmodium ookinetes and are not associated with immune defenses targeting sporozoites or human Plasmodium (Nakhleh et al., 2017). Indeed, the significant abundance of AgCLIPs in uninfected middle-aged An. gambiae may result from agingrelated immune dysfunction. The absence of elevated levels of AgCLIPs highlights another putative 
benefit of Plasmodium-mediated immune priming. Another way to moderate immune effector mechanisms such as melanization is performed by Serpins (serine protease inhibitors). Levels of two Serpins, AgSerpin6 (-0.64-fold; p-adj = <0.0001; AGAP009212) and AgSerpin10 (-0.42; p-adj = 0.03; AGAP005246), that are upregulated in response to Plasmodium ookinetes (Danielli et al., 2003; Abraham et al., 2005) were significantly under-represented compared with uninfected controls (Figure 10C). As melanization is not expected to be active in An. gambiae carrying salivary-gland-stage $P$. falciparum infections, there would be no requirement for Serpin moderation, and such genes as Serpin6 and Serpin10 would no longer be required at this timepoint (Pinto et al., 2007). Likewise, putative immune dysfunction in uninfected controls may impair proper regulation of melanization and, as such, Serpin transcription.

A wide range of immune effectors are downstream of PRR-controlled pathways and other recognition pathways that include anti-microbial peptides (AMPs), reactive oxygen species (ROS), reactive nitrogen species (RNS), prophenoloxidase (PPOs), and apoptosis- and phagocytosis-related genes (Kumar et al., 2018). In Anopheles, five classes of AMPs including attacins, defensins, diptericins, cecropins, and gambicins have been described (Christophides et al., 2002). In our data set, three defensins (Def), three cecropins (Cec), one gambicin (Gam) and one attacin (Att) are present (Figure 10E). Of these, only two defensins and Cec1 were significantly differentially abundant between heads of sporozoite-infected and uninfected control mosquitoes, with AgDef2 (0.96-fold; $p$-adj $=<0.0001$; AGAP004632), AgDef4 (1.96-fold; $p$-adj = 0.005; AGAP005416) and AgCec1 (1.78-fold; $p$-adj = 0.001; AGAP000693) are all significantly abundant in treatment groups compared with controls. Interestingly, these shifts plus the significantly increased abundance of AgGNBPB2 (1.46-fold; $p$-adj = 0.02; AGAP001212; Figure 10A), a PRR that co-localizes $P$. falciparum, are the only other indication 
of an An. gambiae immune response to $P$. falciparum infection in this study. Of these, only AgCec1 is associated with anti-sporozoite activity. AgDef1 and AgDef4 demonstrate antibacterial activity but have not shown any anti-Plasmodium activity (Kokoza et al., 2010). Interestingly, studies in Ae. aegypti have determined that differential Def abundances can be observed without immune challenge, and often after pathogen clearance, suggesting alternative functions for the AMPs (Bartholomay et al., 2004). As such, the role of AgDefs in response to sporozoite infections remains unclear. ROS and RNS are vital effectors of Anopheline immune defenses active against malaria parasites (Kumar et al., 2018; Molina-Cruz et al., 2008; Oliveira et al., 2012). ROS formation in dipterans is postulated to involve NADPH oxidase (NOX)-based production of superoxide anions that are transformed into $\mathrm{H}_{2} \mathrm{O}_{2}$ by superoxide dismutase (SOD) and subsequent production of ROS (Molina-Cruz et al., 2008). NOX is also likely required for heme peroxidase (HPX) and nitric oxide synthase (NOS) activation, both of which potentiate nitration and generations of RNS (Oliveira et al., 2012). Interestingly, the transcripts for all the enzymes associated with ROS/RNS system and their potentiators were largely under-represented in sporozoite-infected head transcriptomes compared with uninfected controls: AgMnSOD1 (-0.88-fold; p-adj = 0.001; AGAP010517), AgCuSOD2 (-0.38fold; $p$-adj = 0.006; AGAP005234), AgHPX2 (-1.20-fold; $p$-adj = <0.0001; AGAP009033) and AgNOXDUOX (-0.63-fold; p-adj = 0.03; AGAP009978; Figure 10E). In that light, it is logical to expect the significant under-representation of transcripts encoding catalase, glutathione peroxidase and thioredoxin peroxidase detoxifying enzymes observed in heads of sporozoite-infected An. gambiae when compared with uninfected controls (Figure 10E).

In Anopheles and other mosquitoes, CLIPs activate PPOs, the primary effectors mediating melanization (Lu et al., 2014). In that light, the lack of differentially abundant AgCLIP transcripts 
aligns with the observation that AgPPOs were predominately under-represented in heads of sporozoite-infected mosquitoes compared with uninfected controls. AgPPO2 (-1.70-fold; $p$-adj = 0.001; AGAP006258), AgPPO5 (-1.30-fold; p-adj = <0.0001; AGAM012616), AgPPO6 (-1.38-fold; padj = 0.002; AGAP004977), and AgPPO9 (-1.50-fold; p-adj = 0.006; AGAP004978) all significantly diminished in An. gambiae carrying salivary-gland-stage sporozoites (Figure 10). The higher PPO levels in heads of uninfected mosquitoes is surprising and may reflect a higher background of metabolic stress. However, this pattern is consistent with the fact that melanized sporozoite-stage Plasmodium has never been observed. In Ae. aegypti, allatostatin neuropeptide interacts with hemocytes and may have subsequently increased AgPPO expression to address dysbiosis and inflammation resulting from IMD destabilization. This finding is particularly interesting because it correlates mosquito immune system fitness with neuropeptide production and has significantly broader implications for aging-related immune system dysfunction. It also provides a plethora of anopheline molecular mechanisms that putatively synergize with Plasmodium immune priming, thereby providing a significant advantage that ultimately facilitates malaria transmission. Apoptosis, autophagy and phagocytosis genes are important effectors in response to viral and pathogen invasion through programmed cell death of infected cells and clearance of cellular debris and pathogen particles (Kumar et al., 2018). In dipterans, apoptosis is induced through the activation of the long caspases, Dronc and Dredd, and inhibition of inhibitors of apoptosis (IAPs) that in turn regulate autophagy genes, with expression of both increasing concurrently (Eng et al., 2016). Transcripts for AgIAP, AgDronc, AgDredd, 18 autophagy-related proteins (AgApgs), and 2 phagocytosis signaling proteins were identified (Figure 10D). AgDronc, AgDredd, and AgIAP were all under-represented in heads of sporozoite-infected mosquitoes when compared with controls, 
although none significantly (Figure 10D). AgApgs were predominately under-represented in treatment groups compared with controls, with only AgApg8 (0.20-fold; $p$-adj $=0.03$; AGAP002685) and AgApg18B (0.59-fold; $p$-adj = 0.003; AGAP005910) significantly abundant in treatment groups, and 8 AgApgs significantly diminished in treatment groups (Figure 10D): AgApg2 (-0.57-fold; p-adj = 0.004; AGAP004092), AgApg3 (-0.69-fold; p-adj = <0.0001; AGAP011582), AgApg6 (-0.37-fold; $p$-adj = 0.02; AGAP003858), AgApg7 (-0.49-fold; $p$-adj = 0.001; AGAP010303), AgApg7A (-1.14-fold; $p$-adj = <0.0001; AGAP008637), AgApg13 (-1.48-fold; $p$-adj = <0.0001; AGAP005715), AgApg16L (-0.67fold; -adj = 0.007; AGAP002315), and AgApg18A (-2.10-fold; p-adj = <0.0001; AGAP007970). Furthermore, the putative phagocytosis signaling proteins, AgActin-5C and AgDSCAM (downsyndrome cell adhesion molecule), were under-represented in treatment transcriptomes, which is not surprising since both AgActin-5C (Sandiford et al., 2015) and AgDSCAM (Dong et al., 2006b) are linked to anti- Plasmodium defense at the midgut-stage infection. Their diminished levels in sporozoite-infected mosquitoes suggest that their active involvement in Plasmodium clearance is unlikely. Indeed, the general overabundance of apoptosis, autophagy, and phagocytosis genes in heads of uninfected control mosquitoes likely reflects the tissue specificity of our study, since phagocytosis is mediated by hemocytes and not epithelial cells, and/or a loss of cell homeostasis that accompanies aging-related immune system dysfunction and again highlights another putative benefit of immune priming in Plasmodium-infected anopheline vectors.

PRRs activate the Toll pathway through cleavage of Spaetzle proteins (SPZs) to activate Dorsal/NKkB transcription factor (Rel1) and regulate antimicrobial peptides (Kumar et al., 2018). The transcriptome profile of these components also supports an aging-related immune system deregulation associated with malarial treatments that is importantly not seen in uninfected controls. 
Transcripts encoding AgRel1 (-0.44-fold; p-adj = 0.002; AGAM009515) along with one Spaeztle, AgSPZ6 (-0.80-fold; p-adj = 0.002; AGAP005126), are both significantly under-represented in heads of sporozoite-infected mosquitoes (Figure 11A). Inasmuch as the Toll pathway has not been linked to defenses against the human malaria parasite P. falciparum (Garver et al., 2009; Dong et al., 2011), it is not surprising that the majority of Toll pathway genes were also significantly under-represented in treatments compared with uninfected controls (Figure 11A). In aging Drosophila, Toll signaling becomes increasingly dysfunctional, causing inflammation, systemic organismal defects, and metabolic deficiencies that result in shortened lifespans (Garschall and Flatt, 2018). In that light, decreased activation of Toll in the heads of sporozoite-infected mosquitoes is part of what we propose to be a broadly synergistic Anopheles-Plasmodium paradigm.

As is the case for Toll-mediated signaling, the anopheline IMD immune signaling pathway involves binding of the PRR, PGRP-LC, which triggers activation of NK-kB transcription factor (Rel2) and production of AMPs. Transcripts for all components of IMD signaling, including PGRP-LC, were identified in sequencing libraries, although AgPGRP-LC (-0.26-fold; $p$-adj = 0.02; AGAP005203) was the sole transcript significantly diminished in heads of sporozoite-infected mosquitoes (Figure 10A, 11B). Even the modest differential abundance of AgPGRP-LC, which is a positive regulator of IMD signaling and PGRP expression, provides a rationale for the lack of significantly differential IMD signaling pathway transcripts in sporozoite-infected mosquitoes (Figure 11B; Kumar et al., 2018). In any case, the destabilization of anopheline IMD signaling pathway components would putatively render the immune signaling system a poor target for Plasmodium immunological priming, as the long-lasting immune response would be unpredictable and difficult to adapt (Cooper and Eleftherianos, 2017). 
The anopheline JAK-STAT signaling pathway has been implicated to function in a variety of dipteran developmental processes, as well as in antimicrobial, antiparasitic and antiviral immune defenses (Clayton et al., 2014). It is negatively regulated by PIAS and suppressor of cytokine signaling (SOCS; Shuai and Liu, 2003) and induced by the binding of Unpaired (Upd) to Domeless (Dome) to form a receptor dimer that, in turn, activates Janus Kinase (JAK) or its homolog Hopscotch (Hop) and ultimately STAT which triggers the production of genes regulated by the JAK-STAT pathway and NOS (Kumar et al., 2018). Transcripts for AgSOCS (0.58-fold; $p$-adj = 0.008; AGAP029624) and AgUPD3 (2.39-fold; p-adj = 0.007; AGAP013506) were significantly more abundant in treatment groups, and AgDome (-0.33-fold; p-adj = 0.006; AGAP029053), AgHop (-1.26-fold; -adj = 0.003; AGAP008354; JAK homolog) and AgSTAT2 (-0.31-fold; $p$-adj = 0.02; AGAP000099) were significantly under-represented compared with uninfected controls (Figure 11B). As is the case for IMD pathways, anopheline JAK-STAT signaling primarily targets pre-sporozoite stages of Plasmodium, making the lack of JAK-STAT activation in the salivary-gland-stage infected An. gambiae examined here expected (Gupta et al., 2009). This is particularly evident by the significant abundance of JAK-STAT's primary negative regulator AgSOC in heads of sporozoite-infected mosquitoes. Dipteran SOCs deactivate the Hop-Dome dimer, block STAT activation, and target components of the Hop-Dome dimer complex for degradation (Croker et al., 2008). As such, the increased abundance of AgUPD3 in treatment groups may reflect replenishment activity to restore UPD3 levels following $P$. falciparum midgut invasion or simply maintenance of normal UPD3 equilibrium. In Drosophila, immune system deregulation and mitochondrial degeneration significantly increase JAK-STAT expression in response to growing systemic inflammation and oxidative stress associated with aging. Excessive JAK-STAT activation ultimately leads to a loss of tissue 
homeostasis and cell death (Garschall and Flatt, 2018). The active inhibition of JAK-STAT signaling in infected mosquitoes would explain the significantly diminished abundance of AgSTAT2 and AgHop and may also reflect aging-related dysfunction in uninfected An. gambiae controls. As such, the anopheline JAK-STAT signaling pathway may represent a target for Plasmodium priming, reducing the detrimental effects of aging-related immune deregulation thereby synergistically benefiting both parasite and host.

General Metabolism and Aging: Metazoan aging can be characterized by the loss of cellular and tissue homeostasis, deterioration of vital biological processes with negative impacts on signaling pathways that regulate nutrient sensing, metabolic allocation, and the maintenance of healthy cells and tissues that together promote mortality (Maruzs et al., 2019). The insect nutrient-sensing pathway TOR regulates cellular metabolism and growth in response to nutrient flux (Wullschleger et al., 2006). In Drosophila, increased TOR signaling is associated with aging, and the inhibition of TOR significantly extends fly lifespan and overall viability (Bjedov et al., 2010). Both anopheline TOR transcripts-AgTOR (-0.44-fold; $p$-adj $=<0.0001 ;$ AGAP007873) and AgTORC1 (-0.51-fold; $p$-adj $=$ 0.003; AGAP010035)—are significantly diminished in heads of sporozoite-infected An. gambiae compared with naïve controls (Figure 12A). Additionally, a transcript for the TOR kinase inhibitor tuberous sclerosis complex 2 (AgTSC2; -0.46-fold; p-adj = <0.001; AGAP003445), which in Drosophila inhibits TOR signaling and acts on fat bodies as a mechanism of lifespan expansion, was found to be significantly diminished in treatment groups compared with controls (Figure 12A; Bjedov et al., 2010). 
The AMP-activated kinase (AMPK) signaling pathway maintains insect energy homeostasis by regulating ATP consumption and production; it is activated in response to low intracellular AMP levels, prompting ATP re-accumulation by reducing costly anaerobic processes as well as activating phosphofructokinase (Pfk), thereby increasing glycogenolysis (Hardie et al., 2012). Interestingly, the transcripts for both AgAMPK (-0.75-fold; $p$-adj $=<0.0001 ;$ AGAP002686) and AgPfk (-0.33-fold; $p$-adj $=0.05 ;$ AGAP007642) were significantly diminished in heads of sporozoite-infected An. gambiae compared with non-infected mosquitoes (Figure 12B). In contrast, AMPK overabundance in An. stephensi is associated with increased innate immune responses and significant alterations in mitochondrial biogenesis (Oringanje et al., 2021). In aging D. melanogaster, increased levels of AMPK improve tissue homeostasis via an anti-inflammatory mechanism to promote longevity (Ulgherait et al., 2014). It is possible that, compared with treatment transcriptomes, the age-related increased immune response observed in heads of naïve An. gambiae results in reduced nutrient and energy homeostasis. This hypothesis is also supported by the significantly diminished abundance of hepatocyte nuclear factor 3 (AgHNF3; -2.17-fold; $p$-adj $=<0.0001 ;$ AGAP001671) and hepatocyte nuclear factor 4 (AgHNF4; -0.98-fold; p-adj = 0.005; AGAP002155) in heads of sporozoite-infected An. gambiae mosquitoes compared with naïve controls. In Ae. Aegypti, RNAi depletion of HNF4 was shown to result in significant downregulation of transcripts encoding triacylglycerol catabolism, which is essential for fat body metabolism (Wang et al., 2017).

The head transcriptomes of naïve An. gambiae controls provide substantial evidence of a robust endemic sterile inflammatory response as well as decreased mitochondrial function, increased mitochondrial damage and mitophagy. In D. melanogaster, mitochondrial dysfunction is closely 
associated with decreased mitochondrial transcript levels of genes such as cytochrome oxidase C (COX) and cytochrome B1 (CB; Schwarze et al., 1998). Indeed, in heads of sporozoite-infected An. gambiae, transcripts for COX and CB were present at very high levels, significantly more abundant than in uninfected controls (Figure 12C). In general, naïve An. gambiae displayed a significant overabundance of transcripts associated with ubiquitination and autophagy, indicating a higher degree of cell dysfunction than in the treatment group (Figure 12F). For example, PTEN-induced kinase 1 (PINK1), a serine/threonine kinase that specifically targets damaged mitochondria for autophagy, is significantly diminished in Plasmodium-infected An. gambiae compared with noninfectious mosquitoes $(-0.66$-fold; $p$-adj $=0.0004$; AGAP004315) (Figure 12B), which may be an indication of a higher degree of mitochondrial dysfunction in naïve aged mosquitoes (Luckhart and Riehle, 2020).

In insects, the forkhead box $\mathrm{O}$ (FOXO) transcription factor signaling pathway is an important moderator of protein quality control, stress resistance, and lifespan extension pathways (Maruzs et al., 2019). FOXO functions with its transcriptional target translation initiation factor, 4E-binding protein (4E-BP), to moderate proteostasis in Drosophila by removing damaged/abnormal proteins (Demontis and Perrimon, 2010). While Ag4E-BP was not significantly different, AgFOXO (-2.36-fold; $p$-adj = $<0.0001$; AGAP000662) was significantly diminished in heads of sporozoite-infected An. gambiae compared with naïve controls (Figure 12D). Similarly, the heat-shock transcription factor (AgHsTF; 1.00 -fold; $p$-adj $=<0.0001 ;$ AGAP029908) and numerous subsequently induced heat shock protein transcripts (Hsps) were all significantly diminished in treatment groups compared with naïve control An. gambiae (Figure 12E). In dipterans, protein abnormalities resulting from oxidative stress gradually 
increase with age and are associated with an increase in HsTF and Hsp production to combat injury from abnormal and malformed proteins (Tower, 2010). Lastly, consistent with these transcriptome shifts, our analyses have also uncovered several transcripts involved in ubiquitin-mediated proteosome protein degradation pathways, the abundance of which are significantly diminished in sporozoite infected mosquitoes (Figure 12F). When viewed collectively, it is likely these differentially abundant transcripts are indicative of an accumulation of abnormal proteins with detrimental effects in naïve, uninfected mosquitoes that importantly does not occur in age-matched counterparts that harbor salivary-gland-stage $P$. falciparum sporozoites after low intensity, biologically relevant bloodmeal infections.

\section{Conclusions}

The impact of Plasmodium infection on the behavior and physiology of anopheline mosquitoes significantly drives vectorial capacity and this study represents the first comprehensive examination of the effects of biologically relevant Plasmodium sporozoite-stage infections on anopheline vectors. To gain insight into this process in as biologically relevant a manner as possible, we have carried out a set of comprehensive comparative analyses of the transcriptome profile of the head (including sensory appendages) and salivary gland tissues of female anophelines that have undergone lowintensity P. falciparum infections representative of those occurring naturally in disease-endemic regions. To our eyes, the significant reduction in prevalence and other experimental challenges associated with these studies are outweighed by the necessity to examine laboratory-based Plasmodium infections under the most biologically relevant conditions possible. Malaria-infected mosquitoes along with their naive counterparts were subsequently aged, thereby facilitating the 
development of salivary-gland-stage sporozoites. As might be expected, these infections have broad effects on transcript abundance (as a partial proxy for differential gene expression) across a large number of gene families. Here, we have focused on those that impact neuronal function and chemosensation as well as age-related immunity and metabolic homeostasis.

The transcriptome profile shifts we have uncovered reveal a variety of systematic impacts that broadly indicate there is a synergistic advantage for mosquitoes harboring salivary gland sporozoites (and therefore likely to be malarious) that aligns with significant increases in their vectorial capacity (Figure 13). These notably include an expected increase in chemosensory sensitivity that could reasonably lead to more successful host seeking of infectious mosquitoes, along with a surprisingly distinctive transcriptome alteration that collectively aligns with an anti-aging paradigm that would likely provide a distinct advantage to these mosquitoes. When compared with similarly aged, uninfected mosquitoes, the synergistic advantage of naturally relevant malaria infections provides at least a partial rationale for the enduring persistence of Plasmodium pathogens and, indeed, global malaria.

\section{Materials and Methods}

\section{Ethics Statement}

Commercial anonymous human blood provided by the Johns Hopkins Institute Core Facility, Baltimore, MD, was used for parasite cultures and mosquito feeding and as such did not require informed consent. The Johns Hopkins School of Public Health Ethics Committee approved the mosquito rearing protocols used in this study. 
Mosquito Rearing and Plasmodium Infections

An. gambiae s.s. (An. gambiae Keele strain; Ranford-Cartwright et al., 2016) mosquitoes were reared and maintained at $27^{\circ} \mathrm{C}$ and $80 \%$ humidity with a 12-h light:12-h dark cycle at Johns Hopkins School of Public Health insectary (Baltimore, MD) according to standard procedures (Dong et al., 2009). Newly emerged, 0-day-old female An. gambiae were split into nine independent lines (groups; six infected or treatment, and three non-infected or control). All control and treatment groups were given their first and only bloodmeal at 5 days old (Haymer et al., 1997). Treatment groups were fed live $P$. falciparum NF54 wild-type (NF54W) gametocyte cultures at a final gametocytemia of $0.008 \%$ in human blood mix (provided by the Johns Hopkins Institute Core Facility, Baltimore, MD) through glass membrane feeders at $37^{\circ} \mathrm{C}$ for $1 \mathrm{~h}$ (Figure $\left.1 \mathrm{~A}\right)$. Controls were fed non-infected naïve commercial anonymous human blood in parallel. All mosquitoes were starved for 3-6 h prior to maximize blood feeding and engorgement. After feeding, treatment and control lines were independently sorted at $4^{\circ} \mathrm{C}$ and unfed and partially fed mosquitoes were removed and disposed. The remaining fully engorged mosquitoes in treatment and control lines were incubated for 18 days in an insectary maintained at $27^{\circ} \mathrm{C}$ and $80 \%$ relative humidity, with a photoperiod of $12 \mathrm{~h}$ light: $12 \mathrm{~h}$ dark, with dusk and dawn periods of $1 \mathrm{~h}$ each at the beginning of each scotophase. All lines had continuous access to sterile distilled water and a $10 \%$ sucrose/distilled water solution throughout the duration of the study. The infection of $P$. falciparum was confirmed at the oocyst stage by sampling at least 8-10 mosquitoes from each replicate at 8 days post infection (Table 1). In total, 58 randomly selected mosquitoes were dissected in PBS, stained with 0.1\% PBS-buffered mercurochrome (MilliporeSigma, Burlington, MA) and examined under a light-contrast microscope (Olympus Life Science, Waltham, 
MA). At the oocyst stage, P. falciparum infection prevalence of the 58 randomly selected infected mosquitoes was $62 \%$, with a median count of two oocysts per mosquito midgut ( $\mathrm{SEM}=0.33$ ).

\section{Mosquito Dissections and Nucleic Acid Extractions}

After collection, each mosquito in this study was individually stored prior to manual trans-section of heads (including sensory appendages) and thorax including salivary glands, referred to as 'head' or 'head tissues', from body/carcass which were stored for future use (Figure 1B). Total RNAs extracted from the heads of established treatment- and control-line An. gambiae mosquitoes were used for Illumina NovaSeq sequencing. Total DNAs were also extracted from the heads of treated mosquitoes for PCR to validate the $P$. falciparum infection at sporozoite stage in individual mosquitoes. All head tissues were collected from control- and treatment-line mosquitoes immediately following the 18-day incubation period (Figure 1). Dissections of control- and treatment-line mosquito heads were performed over 3 days; each line was handled on a different day to prevent cross contamination. All mosquitoes were handled wearing gloves and all dissection equipment washed with glassware detergent, autoclaved, and decontaminated with RNase Away (MilliporeSigma, Burlington, MA). To eliminate potential hemolymph contamination during mosquito dissections, all equipment was sterilized using $90 \%$ ethanol and decontaminated with RNase Away (MilliporeSigma, Burlington, MA) in between individual mosquito handling. To maintain tissue integrity, dissections were performed on cold-anesthetized mosquitoes, and work limited to one line-treatment or control. For treatment lines, the head with intact salivary glands from one mosquito was dissected into a distinct numbered and

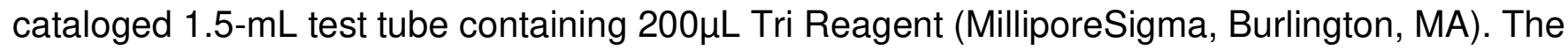
smaller starting volume of Tri Reagent (MilliporeSigma, Burlington, MA) aided subsequent tissue 
homogenization. PCR infection validation studies on treatment-line mosquitoes required that all dissected heads remain separated. Prior to dissections, each treatment-line An. gambiae mosquito was randomly assigned an identification number used for labeling of all associated dissected tissues. In total, the heads of 176 treatment-line mosquitoes were collected into 176 distinct labeled tubes. For control lines, the heads from 30 mosquitoes within a single line were dissected and pooled into one labeled 1.5-mL test tube containing $400 \mu \mathrm{L}$ Tri Reagent (MilliporeSigma, Burlington, MA). This process was repeated for the two remaining control lines. All sample tubes containing dissected head(s) in Tri Reagent (MilliporeSigma, Burlington, MA) were frozen at $-70^{\circ} \mathrm{C}$ in preparation for RNA and DNA extractions.

For RNA extractions, sample tubes were processed in small batches of ten, with treatment and control samples handled separately to prevent cross contamination. A batch of ten sample tubes containing An. gambiae head tissues in Tri Reagent (MilliporeSigma, Burlington, MA) was thawed on ice and the head tissues were homogenized first manually using individual sterile, DNA/RNA-free disposable pellet pestles (Fischer Scientific, Walthom, MA) and second, mechanically by sonication (Q700; QSonica, Newtown, CT) to ensure thorough head tissue and cell disruption. Sonication was performed in short 10 - to 20 -s bursts with samples on ice to minimize nucleic acid shearing. To prevent cross contamination, one pellet pestle was used per sample tube, and the sonicator probe was thoroughly sterilized using 90\% ethanol and decontaminated with RNase Away (MilliporeSigma, Burlington, MA) in between individual sample processing. Following complete tissue homogenization, RNA extractions were performed. An additional 200 $\mathrm{L}$ Tri Reagent (MilliporeSigma, Burlington, MA) was added to each treatment sample tube bringing the total volume to $400 \mu \mathrm{L}$, equivalent to control 
sample volumes. Sample tubes were then hand shaken and incubated at room temperature for $5 \mathrm{~min}$.

A 100- $\mu \mathrm{L}$ aliquot of UltraPure phenol:chloroform:isoamyl alcohol (25:24:1, v/v; Thermo Fischer, Walthom, MA) was added to each of the tubes, which were then vigorously hand shaken for $1 \mathrm{~min}$ and incubated at room temperature for $5 \mathrm{~min}$. For complete phase separation, sample tubes were centrifuged at $13,000 \mathrm{RPM}$ for $15 \mathrm{~min}$ at $4^{\circ} \mathrm{C}$. The resulting aqueous phase (containing RNAs) from each sample tube was carefully transferred into a coordinately labeled new RNase-free 1.5 -mL test tube containing $100 \mu \mathrm{L} 70 \%$ ethanol and thoroughly mixed by pipetting. Sample tubes containing the remaining interphase and organic phase were frozen at $-70^{\circ} \mathrm{C}$ in preparation for DNA extractions. Total RNAs were extracted from the aqueous phase/ethanol solutions according to the manufacturer's protocol using the PureLink RNA Mini Kit (Thermo Fischer, Waltham, MA). This process was repeated for all 179 treatment and control sample tubes. Total RNA concentrations and purities were measured using NanoDrop (Thermo Fischer, Waltham, MA) and any detected phenol contaminants removed by 1-butanol extraction (Krebs et al., 2009). Control-sample total RNAs were kept separate to allow for three distinct biological replicates pertinent to RNAseq analyses. For the treatment replicates, after each individual sample was confirmed to contain $P$. falciparum sporozoites via PCR validation, three distinct pools were prepared for RNAseq analyses. Ultimately, 65 of the 176 treatment samples were positively confirmed for $P$. falciparum infection and total RNAs were pooled into three replicates (21 samples per replicate).

DNA extractions were performed on all treatment sample tubes containing remaining interphase and organic phase solutions to perform PCR P. falciparum validation studies. Like RNA extractions, treatment samples were processed in small batches of ten to preserve DNA integrity. A batch of 
treatment sample tubes was thawed on ice, centrifuged at 13,000 RPM for 5 min at $4^{\circ} \mathrm{C}$, and any remaining aqueous phase was removed and disposed of to prevent RNA contamination of DNA extracts. Then, $200 \mu \mathrm{L}$ back extraction buffer was added to each sample tube and hand mixed by inversion for $3 \mathrm{~min}$. For complete phase separation, sample tubes were centrifuged at 13,000 RPM for $30 \mathrm{~min}$ at $4^{\circ} \mathrm{C}$. The resulting aqueous phase (containing DNAs) from each sample was carefully transferred into a coordinately labeled, new sterile $1.5-\mathrm{mL}$ test tube containing $200 \mu \mathrm{L} 70 \%$ ethanol and thoroughly mixed by pipetting. Total DNAs were extracted from the aqueous phase/ethanol solutions according to the manufacturer's protocol using the DNAEasy Blood and Tissue Kit (Qiagen, Germantown, MD). Total DNA concentrations and purities were measured using NanoDrop (Thermo Fischer, Walthom, MA) and any detected phenol contaminants removed by 1 -butanol extraction (Krebs et al., 2009). The total gDNA was finally resuspended at a final concentration of $10 \mathrm{ng} / \mu \mathrm{L}$ and kept at $-20^{\circ} \mathrm{C}$ for further PCR experiments.

\section{PCR-Based Plasmodium infection validation}

PCR experiments were performed to validate the presence of $P$. falciparum sporozoites within each treatment sample. PCR experiments used total DNAs extracted from dissected heads and salivary glands of individual treatment-line An. gambiae female mosquitoes (Ref. Mosquito Dissections and Nucleic Acid Extractions). PCR was performed using an MJ Thermal Cycler (BioRad Inc, Hercules, CA) in combination with the DreamTaq Mastermix@ technology (Thermo Scientific, Waltham, MA) according to the manufacturer's protocol. PCR products were visualized on a $1 \%$ agarose gel using the Quick-load Purple 1-Kb DNA ladder (New England Biolabs, Ipswich, MA). Primer3 Plus (Untergasser et al., 2012) was used to design primer pairs for the $P$. falciparum circumsporozoite 
gene (CS) and the An. gambiae ribosomal s7 gene (AgRsp7) used as a reference for internal standardization (Coppi et al., 2011). The primer sets used were as follows:

CS forward (Fw): 5'-ACGAGAAATTAAGGAAACCAAAACA- 3'.

CS reverse (Rv): 5' -ACCTTGTCCATTACCTTGATTGT- 3'.

AgRspTFw: 5' -AGAATCGAACTCTGGTGGCTG- 3.

AgRsp4Rv: 5' -ACACAACATCGAAGGATACGA- 3'.

All PCR primers were validated and optimized in preliminary studies. PCR experiments were conducted for all treatment samples, with a correlating negative control (blank) and positive $P$. falciparum infected positive control (Figure 2). Treatment samples producing CS PCR products were reconfirmed to be positively infected through a second follow-up PCR experiment.

\section{Illumina NovaSeq Paired-end Sequencing}

Deep sequencing utilizing Illumina RNAseq technology (Illumina, San Diego, CA) was performed at Hudson Alpha Discovery Genomic Services Laboratory (Huntsville, AL). Poly(A) mRNAs were isolated separately for library preparation and barcoding from approximately 500ng of total RNA collected from each of the three control replicates and approximately 300ng of total RNA collected from each of the three treatment replicates. Final mRNAs were fragmented, and cDNA was synthesized, amplified, digested, and purified using Illumina TruSeq chemistry protocols (Illumina, San Diego, CA). In preparation for sequencing, each of the six libraries-three treatment and three controls-was labelled with a 16-bp barcode to identify sequencing data generated from each library [treatment replicate $1(\mathrm{~T} 1)=$ TTGCAGGTTGCAGCGG, treatment T2 = CAACTGTATGAGTGCC, treatment T3 = AGTCAATGTAGAGGCC, control C1 = ACAAGCTTACAACTAT, control C2 = 
CGTCCTAACCATCGGC, and control C3 = TTGAGAACTCTCTAAC]. Barcoded treatment and control replicate libraries were hybridized across four lanes of two S4 flow cells for cBot (Illumina, San Diego, CA) cluster generation and sequencing (paired-end, $2 \times 150 \mathrm{bp}$; total 300 cycles). A total of 36 raw data sets of sequencing outputs, 6 per library, were generated using the Illumina software assembler (Illumina, San Diego, CA).

\section{Illumina Bioinformatics}

The 36 control and treatment replicate Illumina NovaSeq data sets generated were cleaned and quality trimmed (Q30) using Trimmomatic v0.33 (Bolger et al., 2014), then verified for quality control using FastQC v.0.11.9 (https://www.bioinformatics.babraham.ac.uk/projects/fastqc/). Control and treatment replicate data sets were aligned to the An. gambiae PEST strain genome (Vectorbase53_AgambiaePEST_Genome.fa, https://vectorbase.org) using STAR v.2.7.5

(https://github.com/alexdobin/STAR), with An. gambiae PEST strain gene annotations (Vectorbase53_AgambiaePEST.gff, https://vectorbase.org) to map splice junctions, 2-pass mapping, -sjdbOverhang set to $149 \mathrm{bp}, \mathrm{MAPQ}$ set to 60 , --align IntronMin set to $10 \mathrm{bp}$, and a preliminary UNIX concatenate script resulting in six output BAM alignment files: control R1, control R2, control R3, treatment R1, treatment R2, and treatment R3. HTSeq v0.11.2 (Anders et al., 2015), an alignmentbased count algorithm, was used to quantify transcript counts for all six BAM alignment files with default parameters and mapping validation. DESeq2 (Bioconductor v.3.8) and R v.3.5.1 were used to calculate mean variance and obtain differentially abundant transcripts between control and treatment replicates using default parameters with added shrinkage and Wald's hypothesis tests. Gene ontologies (GO) of differentially abundant transcripts were obtained from OmicsBox using the 
GenBank non-redundant protein (nr) database containing all available mosquito genomes with an expected value (e-value) cutoff of less than 0.01 . All GO annotations were thereafter manually confirmed via BLAST, and phylogenetic analyses and FPKM values for differentially abundant transcripts were analyzed for significance using ANOVA with Sidak's multiple comparison test using Prism $^{\text {TM }}$ v.8 (GraphPad, La Jolla, CA).

\section{Figure Legends}

Figure 1. Schematic representation of experimental methodology. (A) Timeline for establishment of Plasmodium falciparum sporozoite infected treatment and naive controls An. gambiae s.s. mosquito lines. (B) Total RNA and gDNA extraction treatment and control protocols for PCR P. falciparum sporozoite infection validation and RNA sequencing.

Figure 2. (A) P. falciparum circumsporozoite (CS) primer set schematics. (B) Agarose gel (1.5\%) electrophoresis image of amplified products using CS2 primer sets. Lanes 1-6 and 10-16 are examined $P$. falciparum sporozoite infected treatment $A n$. gambiae s.s. mosquitoes with a (Kazuya Ujihara) positive and (-) negative control. Lane Ld, 100bp DNA size marker.

Figure 3. (A) Scatterplot of log fold changes vs. mean normalized counts generated using HTSeq and DESeq2 for $P$. falciparum sporozoite infected treatment and naïve control library replicates $(p<$ 0.05). (B) Principal component analysis of the 3 sporozoite infected treatment and 3 naïve control library replicates. Gray circles represent control replicates; blue circles represent treatment replicates.

Figure 4. Distribution of transcripts annotated at the gene ontology level 2 and their putative involved in biological functions for $(\mathrm{A})$ all transcripts differentially abundant between $P$. falciparum sporozoite 
infected treatment An. gambiae s.s. and naïve controls, (B) only transcripts differentially abundant in sporozoite infected treatments, and (C) only transcripts differentially abundant in naïve controls.

Figure 5. Chemosensory differential transcript abundances following relevant sporozoite infections. Chemosensory transcripts that were present at significantly higher (blue) or lower (yellow) levels in $P$. falciparum sporozoite infected treatments; non-differentially expressed chemosensory transcripts are denoted as zeros (black). Chemosensory genes within each family organized by adjusted p-value and subsequently arrayed left to right from most abundant to least abundant based on FPKM values (quartile bars above each image). (A) Odorant receptor family (OR). (B) Odorant binding protein family (Pattanakitsakul et al.). Chemosensory co-receptors are identified by red boxes. Log 2 scale indicates transcript abundances that were significantly higher (blue) or lower (yellow) in sporozoite infected treatments (Vrba et al.) vs. naïve controls (Cnt).

Figure 6. Calculated changes in AgOR mediated odorant receptivity following relevant sporozoite infections. Graphic representation of conceptualized differences in odorant receptivity for 70 odors in P. falciparum sporozoite infected treatments (Vrba et al.) vs. naïve controls (Cnt). Response characteristics were determined using known An. gambiae odorant receptor responses in heterologous expression systems and weighted by relative AgOR expression levels for each corresponding receptor. Results are sorted high to low. Scale bar show calculated increases (blue).

Figure 7. Chemosensory differential transcript abundances following relevant sporozoite infections. Chemosensory transcripts that were present at significantly higher (blue) or lower (yellow) levels in $P$. falciparum sporozoite infected treatments; non-differentially expressed chemosensory transcripts are denoted as zeros (black). Chemosensory genes within each family organized by adjusted $p$-value and subsequently arrayed left to right from most abundant to least abundant based on FPKM values 
(quartile bars above each image). (A) Gustatory receptor family (GR). (B) lonotropic receptor family (IR). (C) Transient receptor protein family (Trpis et al.). (D) Pickpocket protein family (Thiery et al.). (E) Sensory neuron membrane protein family (SNMP). Chemosensory co-receptors are identified by red boxes. The three $\mathrm{CO}_{2}$ gustatory receptors are identified by a red line. $\mathrm{Log}_{2}$ scale indicates transcript abundances that were significantly higher (blue) or lower (yellow) in sporozoite infected treatments (Vrba et al.) vs. naïve controls (Cnt).

Figure 8. Neurotransmitter differential transcript abundances following relevant sporozoite infections. Neurotransmitter transcripts that were present at significantly higher (blue) or lower (yellow) levels in P. falciparum sporozoite infected treatments; non-differentially expressed neurotransmitter transcripts are denoted as zeros (black). Neurotransmitter genes within each family organized by adjusted pvalue and subsequently arrayed left to right from most abundant to least abundant based on FPKM values (quartile bars above each image). (A) Acetylcholine receptor family. (B) Gamma aminobutyric acid receptor family. (C) Glutamate receptor family. (D) Histamine receptor family. (E) Serotonin receptor family. (F) Octopamine receptor family. (G) Dopamine receptor family. Log2 scale indicates transcript abundances that were significantly higher (blue) or lower (yellow) in sporozoite infected treatments (Vrba et al.) vs. naïve controls (Cnt).

Figure 9. Neuropeptide differential transcript abundances following relevant sporozoite infections. Neuropeptide transcripts that were present at significantly higher (blue) or lower (yellow) levels in $P$. falciparum sporozoite infected treatments; non-differentially expressed neuropeptide transcripts are denoted as zeros (black). Neuropeptide genes within each family organized by adjusted p-value and subsequently arrayed left to right from most abundant to least abundant based on FPKM values (quartile bars above each image). (A) Neurotransmitter processing enzyme family. (B) Neuropeptide 
family. (C) Neuropeptide receptor family. (D) Neuron transporter family. $\log _{2}$ scale indicates transcript abundances that were significantly higher (blue) or lower (yellow) in sporozoite infected treatments (Vrba et al.) vs. naïve controls (Cnt).

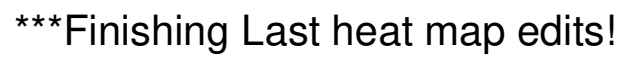

Figure 10. Immune response differential transcript abundances following relevant sporozoite infections. Immune response transcripts that were present at significantly higher (blue) or lower (yellow) levels in P. falciparum sporozoite infected treatments; non-differentially expressed immune response transcripts are denoted as zeros (black). Immune response genes within each group organized by adjusted $p$-value and subsequently arrayed left to right from most abundant to least abundant based on FPKM values (quartile bars above each image). (A-B) Pattern recognition proteins. (C) Modulator proteins. (D-E) Effector proteins. Log 2 scale indicates transcript abundances that were significantly higher (blue) or lower (yellow) in sporozoite infected treatments (Vrba et al.) vs. naïve controls (Cnt).

Figure 11. Immune response differential transcript abundances following relevant sporozoite infections. Immune response transcripts that were present at significantly higher (blue) or lower (yellow) levels in $P$. falciparum sporozoite infected treatments; non-differentially expressed immune response transcripts are denoted as zeros (black). Immune response genes within each group organized by adjusted p-value and subsequently arrayed left to right from most abundant to least abundant based on FPKM values (quartile bars above each image). (A) Toll and spaetzle proteins. (B). IMD and JAK-STAT proteins. Log $_{2}$ scale indicates transcript abundances that were significantly higher (blue) or lower (yellow) in sporozoite infected treatments (Vrba et al.) vs. naïve controls (Cnt). 
Figure 12. Aging biomarker differential transcript abundances following relevant sporozoite infections. Aging biomarker transcripts that were present at significantly higher (blue) or lower (yellow) levels in P. falciparum sporozoite infected treatments; non-differentially expressed Aging biomarker transcripts are denoted as zeros (black). Aging biomarker genes within each group organized by adjusted pvalue and subsequently arrayed left to right from most abundant to least abundant based on FPKM values (quartile bars above each image). (A) TOR and rapamycin proteins. (B). AMPK proteins. (C) Cytochrome C and B proteins. (D) FOXO proteins. (E) Heat shock proteins. (F) Ubiquitination proteins. $\log _{2}$ scale indicates transcript abundances that were significantly higher (blue) or lower (yellow) in sporozoite infected treatments (Vrba et al.) vs. naïve controls (Cnt).

Figure 13. Schematic depicting transcriptional shifts in An. gambiaei following infection with $P$. falciparum sporozoites compared to naïve controls. Text is weighted to emphasize degree of transcript abundance change.

Table 1. Results from midgut oocyst counts of 8-d.o. P. falciparum infected treatment An. gambiae s.s. mosquitoes.

Table 2. Statistics of RNA sequencing for $P$. falciparum sporozoite infected treatment and naïve control An. gambiae s.s. libraries.

Figure S1. (A) Schematic representation of gDNA extraction methodology from head and salivary gland tissues of $P$. falciparum sporozoite infected treatment mosquitoes. (B) Agarose gel (1.5\%) electrophoresis image of amplified products using CS2 primer sets. Lanes $1 \mathrm{Sg}$ and $1 \mathrm{H}$ represent examined salivary gland and head tissues, respectively from treatment An. gambiae s.s. (An. gambiae) mosquito 1. Remaining lanes follow a like organization for 5 additional treatment $A n$. 
gambiae mosquitoes with a (Kazuya Ujihara) positive and (-) negative control. Lane Ld, 100bp DNA size marker.

\section{Acknowledgments}

We thank Ms. Maureen Ubani for technical assistance for PCR validations; Dr. Antonis Rokas, Dr. H. W. Honegger and Dr. Stephen Ferguson for their comments on this manuscript as well as other members of the Zwiebel lab for critical suggestions during the course of this work. We also thank Dr.

A.M. McAinsh for editorial assistance as well as the Johns Hopkins Malaria Research Institute insectary and parasitology core facility, for mosquito rearing and other technical help. This work was conducted with the support of the Bloomberg Philanthropies (to G.D.), Vanderbilt University (to LJZ) and National Institutes of Health grants (NIAID, Al122743) to GD, and (NIAID, Al127693) to LJZ.

\section{Author Contributions:}

A.L.C. and L.J.Z. designed the study. A.L.C. and Y. D. performed the experiments. A.L.C and D.R. analyzed data and prepared figures; A.L.C, D.R., Y.D., G.D. and L.J.Z. interpreted the results and wrote the manuscript.

\section{Declaration of Interests}

The authors declare no competing interests. 


\section{References}

Abraham, E.G., Pinto, S.B., Ghosh, A., Vanlandingham, D.L., Budd, A., Higgs, S., Kafatos, F.C., Jacobs-Lorena, M., and Michel, K. (2005). An immune-responsive serpin, SRPN6, mediates mosquito defense against malaria parasites. Proc Natl Acad Sci U S A 102, 16327-16332.

Aguilar, R., Jedlicka, A.E., Mintz, M., Mahairaki, V., Scott, A.L., and Dimopoulos, G. (2005). Global gene expression analysis of Anopheles gambiae responses to microbial challenge. Insect Biochem Mol Biol 35, 709-719. 10.1016/j.ibmb.2005.02.019.

Anders, S., Pyl, P.T., and Huber, W. (2015). HTSeq--a Python framework to work with highthroughput sequencing data. Bioinformatics 31, 166-169. doi: 10.1093/bioinformatics/btu638.

Anderson, R.A., Koella, J.C., and Hurd, H. (1999). The effect of Plasmodium yoelii nigeriensis infection on the feeding persistence of Anopheles stephensi Liston throughout the sporogonic cycle. Proceedings Biological sciences / The Royal Society 266, 1729-1733. 10.1098/rspb.1999.0839.

Araujo, D.M., Lapchak, O.A., Meaney, M.J., Collier, B., and Quirion, R. (1990). Effects of aging on nicotinic and muscarinic autoreceptor function in the rat brain: relationship to presynaptic cholinergic markers and binding sites. J Neurosci 10, 2069-3078. 
Badsha, F., Kain, P., Prabhakar, S., Sundaram, S., Padinjat, R., Rodrigues, V., and Hasan, G. (2012). Mutants in Drosophila TRPC channels reduce olfactory sensitivity to carbon dioxide. PLoS One 7, e49848. 10.1371/journal.pone.0049848.

Bartholomay, L.C., Fuchs, J.F., Cheng, L.-L., Beck, E.T., Vizioli, J., Lowenberger, C., and Christensen, B.M. (2004). Reassessing the role of defensin in the innate immune response of the mosquito, Aedes aegypti. Insect Mol Biol 13, 125-132. 10.1111/j.0962-1075.2004.00467.x.

Belachew, E.B. (2018). Immune response and evasion mechanisms of Plasmodium falciparum parasites. Journal of Immunology Research 2018, 6529681. https://doi.org/10.1155/2018/6529681.

Bjedov, I., Toivonen, J.M., Kerr, F., Jacobson, J., Foley, A., and Patridge, L. (2010). Mechanisms of life span extension by rapamycin in the fruit fly Drosophila melanogaster. Cell Metab 11, 35-46. 10.1016/j.cmet.2009.11.010.

Blandin, S., Shiao, S.H., Moita, L.F., Janse, C.J., Waters, A.P., Kafatos, F.C., and Levashina, E.A. (2004). Complement-like protein TEP1 is a determinant of vectorial capacity in the malaria vector Anopheles gambiae. Cell 116, 661-670.

Bohbot, J.D., Sparks, J.T., and Dickens, J.C. (2014). The maxillary palp of Aedes aegypti, a model of multisensory integration. Insect Biochem Mol Biol 48, 29-39. 10.1016/j.ibmb.2014.02.007. 
Bolger, A.M., Lohse, M., and Usadel, B. (2014). Trimmomatic: a flexible trimmer for Illumina sequence data. Bioinformatics 30, 2114-2120. 10.1093/bioinformatics/btu170.

Cassau, S., and Krieger, J. (2020). The role of SNMPs in insect olfaction. Cell Tiss Res 383, 21-33. 10.1007/s00441-020-03336-0.

Cator, L.J., Lynch, P.A., Thomas, M.B., and Read, A.F. (2014). Alterations in mosquito behaviour by malaria parasites: potential impact on force of infection. Malaria Journal 13, 164 doi:

$10.1017 / \mathrm{S} 0031182000085127$.

Christophides, G.K., Zdobnov, E., Barillas-Mury, C., Birney, E., Blandin, S., Blass, C., Brey, P.T., Collins, F.H., Danielli, A., Dimopoulos, G., et al. (2002). Immunity-related genes and gene families in Anopheles gambiae. Science 298, 159-165. 10.1126/science.1077136 298/5591/159 [pii].

Clayton, A.M., Dong, Y., and Dimopoulos, G. (2014). The Anopheles innate immune system in the defense against malaria infection. J Innate Immun 6, 169-181. 10.1159/000353602.

Coetzee, M., and Koekemoer, L.L. (2013). Molecular Systematics and Insecticide Resistance in the Major African Malaria Vector Anopheles funestus. Annual review of entomology 58, 393-412. 10.1146/annurev-ento-120811-153628. 
Collins, F.H., and Besansky, N.J. (1994). Vector biology and the control of malaria in Africa. Science $264,1874-1875$.

Cooper, D., and Eleftherianos, I. (2017). Memory and Specificity in the Insect Immune System: Current Perspectives and Future Challenges. Front Immunol 8. 10.3389/fimmu.2017.00539.

Coppi, A., Natarajan, R., Pradel, G., Bennett, B.L., James, E.R., Roggero, M.A., Corradin, G., Persson, C., Tewari, R., and Sinnis, P. (2011). The malaria circumsporozoite protein has two functional domains, each with distinct roles as sporozoites journey from mosquito to mammalian host. J Exp Med 208, 341-356. doi: 10.1084/jem.20101488.

Cork, A., and Park, K.C. (1996). Identification of electrophysiologically-active compounds for the malaria mosquito, Anopheles gambiae, in human sweat extracts. Med Vet Entomol 10, 269-276.

Cribellier, A., Spitzen, J., Fairbairn, H., van de Geer, C., van Leeuwen, J.L., and Muijres, F.T. (2020). Lure, retain, and catch malaria mosquitoes. How heat and humidity improve odour-baited trap performance. Malar J 19, 357. 10.1186/s12936-020-03403-5.

Croker, B.A., Kiu, H., and Nicholson, S.E. (2008). SOCS regulation of the JAK/STAT signalling pathway. Semin Cell Dev Biol 19, 414-422. 10.1016/j.semcdb.2008.07.010. 
Croset, V., Rytz, R., Cummins, S.F., Budd, A., Brawand, D., Kaessmann, H., Gibson, T.J., and Benton, R. (2010). Ancient protostome origin of chemosensory ionotropic glutamate receptors and the evolution of insect taste and olfaction. PLoS genetics 6, e1001064. 10.1371/journal.pgen.1001064.

Danielli, A., Kafatos, F.C., and Loukeris, T.G. (2003). Cloning and characterization of four Anopheles gambiae serpin isoforms, differentially induced in the midgut by Plasmodium berghei invasion. J Biol Chem 278, 4184-4193.

Demontis, F., and Perrimon, N. (2010). FOXO/4E-BP signaling in Drosophila muscles regulates organism-wide proteostasis during aging. Cell 143, 813-825. 10.1016/j.cell.2010.10.007.

Dhara, A., Eum, J.-H., Robertson, A., Gulia-Nuss, M., Vogel, K.J., Clark, K.D., Graf, R., Brown, M.R., and Strand, M.R. (2013). Ovary ecdysteroidogenic hormone functions independently of the insulin receptor in the yellow fever mosquito, Aedes aegypti. Insect biochemistry and molecular biology 43 , 1100-1108. 10.1016/j.ibmb.2013.09.004.

Dong, Y., Aguilar, R., Xi, Z., Warr, E., Mongin, E., and Dimopoulos, G. (2006a). Anopheles gambiae immune response to human and rodent Plasmodium parasite species. PLoS Pathog 2, e52. 10.1371/journal.ppat.0020052. 
Dong, Y., Manfredini, F., and Dimopoulos, G. (2009). Implication of the mosquito midgut microbiota in the defense against malaria parasites. PLoS Pathog. 5, e1000423. 10.1371/journal.ppat.1000423.

Dong, Y., Taylor, H.E., and Dimopoulos, G. (2006b). AgDscam, a Hypervariable Immunoglobulin Domain-Containing Receptor of the Anopheles gambiae Innate Immune System. PLoS Biol 4, e229. 10.1371/journal.pbio.0040229.

Eng, M.W., van Zuylen, M.N., and Severson, D.W. (2016). Apoptosis-related genes control autophagy and influence DENV-2 infection in the mosquito vector, Aedes aegypti. Insect Biochem Mol Biol 76, 70-83. 10.1016/j.ibmb.2016.07.004.

Ferguson, H.M., and Read, A.F. (2004). Mosquito appetite for blood is stimulated by Plasmodium chabaudi infections in themselves and their vertebrate hosts. Malaria Journal 3, 3-12.

Fowler, M.A., and Montell, C. (2013). Drosophila TRP channels and animal behavior. Life Sci. 92, 394-403.

Freeman, E.G., and Dahanukar, A. (2015). Molecular neurobiology of Drosophila taste. Curr Opin Neurobiol 34, 140-148. 10.1016/j.conb.2015.06.001.

Garschall, K., and Flatt, T. (2018). The interplay between immunity and aging in Drosophila. F1000Red 7, 160. 10.12688/f1000research.13117.1. 
Gary, R.E., Jr., and Foster, W.A. (2001). Effects of available sugar on the reproductive fitness and vectorial capacity of the malaria vector Anopheles gambiae (Diptera: Culicidae). J Med Entomol 38, 22-28.

Grant, A.J., Muskavitch, M.A.T., and O'Connell, R.J. (2013). Malaria infection does not affect the sensitivity of peripheral receptor neurons in Anopheles stephensi. Parasites \&amp; Vectors 6, 134. 10.1038/474040a.

Guo, L., Karpac, J., Tran, S.L., and Jasper, H. (2014). PGRP-SC2 Promotes gut immune homeostasis to limit commensal dysbiosis and extend lifespan. Cell 156, 109-122. https://doi.org/10.1016/j.cell.2013.12.018.

Gupta, L., Molina-Cruz, A., Kumar, S., Rodrigues, J., Dixit, R., Zamora, R.E., and Barillas-Mury, C. (2009). The STAT pathway mediates late-phase immunity against Plasmodium in the mosquito Anopheles gambiae. Cell Host Microbe 5, 498-507. 10.1016/j.chom.2009.04.003.

Hagan, R.W., Didion, E. M., Rosselot, A. E., Holmes, C. J., Siler, S.C., Rosendale, A. J., Hendershot, J. M., Elliot, K. S. B., Jennings, E. C., Nine, G. A., Perez, P. L., Rizlallah, A. E., Watanabe, M., 
Romick-Rosendale, L. E., Xioa, Y. Rasgon, J. L.., and Benoit, J. B. (2018). Dehydration prompts increased activity and blood feeding by mosquitoes. Scientific Reports 8. 10.1038/s41598-018-24893-

Z.

Hardie, D.G., Ross, F.A., and Hawley, S.A. (2012). AMPK: A nutrient and energy sensor that maintains energy homeostasis. Nat Rev Mol Cell Biol 13, 251-262. 10.1038/nrm3311.

Haymer, D.S., He, M., and D.O., M. (1997). Genetic marker analysis of spatial and temporal relationships among existing populations of the Mediterranean fruit fly. Heredity $79,302-309$.

Hernandez-Martinez, S., Sanchez-Zavaleta, M., Brito, K., Herrera-Ortiz, A., Ons, S., and Noriega, F.G. (2017). Allatotropin: A pleiotropic neuropeptide that elicits mosquito immune responses. PLoS One 12, e0175759. 10.1371/journal.pone.0175759.

Hill, S.R., Ghaninia, M., and Ignell, R. (2019). Blood meal induced regulation of gene expression in the maxillary palps, a chemosensory organ of the mosquito Aedes aegypti. Front Ecol Evol 10. https://doi.org/10.3389/fevo.2019.00336.

Hodo, T.W., de Aquino, M.T.P., Shinamoto, A., and Shanker, A. (2020). Critical neurotransmitters in the neuroimmune network. Front Immunol 11, 1869. 10.3389/fimmu.2020.01869. 
Huestis, D.L., Artis, M.L., Armbruster, P.A., and Lehmann, T. (2017). Photoperiodic responses of Sahelian malaria mosquitoes Anopheles coluzzii and An. arabiensis. Parasite Vector 10, 621. https://doi.org/10.1186/s13071-017-2556-z.

Karner, T., Kellner, I., Schultze, A., Breer, H., and Krieger, J. (2015). Co-expression of six tightly clustered odorant receptor genes in the antenna of the malaria mosquito Anopheles gambiae. Frontiers in Ecology and Evolution 3. 10.3389/fevo.2015.00026.

Katewa, S.D., and Kapahi, P. (2010). Role of TOR signaling in aging and related biological processes in Drosophila melanogaster. Exp Gerontol 46, 382-390. 10.1016/j.exger.2010.11.036.

Kazuya Ujihara, T.w.c.s.b.a.F.--.-E.-m.u.s.s.-c.c.j.T.M., Tomonori Iwasaki, Masayo Sugano, Yoshinori Shono, and Noritada Matsuo (2004). Metofluthrin: A Potent New Synthetic Pyrethroid with High Vapor Activity against Mosquitoes.

Kent, L.B., Walden, K.K., and Robertson, H.M. (2008). The Gr family of candidate gustatory and olfactory receptors in the yellow-fever mosquito Aedes aegypti. Chem. Senses 33, 79-93.

Kokoza, V., Ahmed, A., Woon Shin, S., Okafor, N., Zou, Z., and Raikhel, A.S. (2010). Blocking of Plasmodium transmission by cooperative action of Cecropin A and Defensin A in transgenic Aedes aegypti mosquitoes. Proceedings of the National Academy of Sciences 107, 8111-8116.

10.1073/pnas.1003056107. 
Krebs, S., Fischaleck, M., and Blum, H. (2009). A simple and loss-free method to remove TRIzol contaminations from minute RNA samples. Anal Biochem 387, 136-138. doi: 10.1016/j.ab.2008.12.020.

Kumar, A., Srivastava, P., Sirisena, P., Dubey, S.K., Kumar, R., Shrinet, J., and Sunil, S. (2018). Mosquito Innate Immunity. Insects 9, 95. 10.3390/insects9030095.

Lacroix, R., Mukabana, W.R., Gouagna, L.C., and Koella, J.C. (2005). Malaria infection increases attractiveness of humans to mosquitoes. PLOS Biology 3, e298.

Leal, W.S. (2013). Odorant reception in insects: roles of receptors, binding proteins, and degrading enzymes. Annu. Rev. Entomol. 58, 373-391. 10.1146/annurev-ento-120811-153635.

Lee, K., Kwon, O., Lee, J.H., Kwon, K., Min, K., Jung, S., Kim, A., You, K., Tatar, M., and Yu, K. (2008). Drosophila short neuropeptide F signalling regulates growth by ERK-mediated insulin signalling. Nat Cell Biol 10, 468-475. 10.1038/ncb1710.

Lenz, O., Xiong, J., Nelson, M.D., Raizen, D.M., and Williams, J.A. (2015). FMRFamide signaling promotes stress-induced sleep in Drosophila. Brain Behav Immun 47, 141-148.

10.1016/j.bbi.2014.12.028. 
Liu, C., Pitts, R.J., Bohbot, J.D., Jones, P.L., Wang, G., and Zwiebel, L.J. (2010). Distinct olfactory signaling mechanisms in the malaria vector mosquito Anopheles gambiae. PLoS Biol 8.

10.1371/journal.pbio.1000467.

Liu, F., Ye, Z., Baker, A., Sun, H., and Zwiebel, L.J. (2020). Gene editing reveals obligate and modulatory components of the $\mathrm{CO} 2$ receptor complex in the malaria vector mosquito, Anopheles coluzzii. Insect Biochem Mol Biol 127, 103470. 10.1016/j.ibmb.2020.103470.

Lu, A., Zhang, Q., Zhang, J., Yang, B., Wu, K., Luan, Y., and Ling, E. (2014). Insect prophenoloxidase: the view beyound immunity. Front Physoil 5. 10.3389/fphys.2014.00252.

Luckhart, S., and Riehle, M.A. (2020). Midgut mitochondrial function as a gatekeeper for malaria parasite infection and development in the mosquito host. Front Cell Infect Microbiol 10, 593159. 10.3389/fcimb.2020.593159.

malERA (2011). A research agenda for malaria eradication: Cross-cutting issues for eradication. PLoS Med 8, e1000404.

Maruzs, T., Simon-Vecsei, Z., Kiss, V., Csizmadia, T., and Juhasz, G. (2019). On the fly: recent progress on autophagy and aging in Drosophila. Front Cell Dev Biol. https://doi.org/10.3389/fcell.2019.00140. 
Matthews, B.J., McBride, C.S., DeGennaro, M., Despo, O., and Vosshall, L.B. (2016). The neurotranscriptome of the Aedes aegypti mosquito. BMC Genomics 17, 32. 10.1186/s12864-0152239-0.

Menuz, K., Larter, N.K., Park, J., and Carlson, J.R. (2014). An RNA-Seq Screen of the Drosophila Antenna Identifies a Transporter Necessary for Ammonia Detection. Plos Genet 10, e1004810. 10.1371/journal.pgen.1004810.s010.

Molina-Cruz, A., DeJong, R.J., Charles, B., Gupta, L., Kumar, S., Jaramillo-Gutierrez, G., and Barillas-Mury, C. (2008). Reactive oxygen species modulate Anopheles gambiae immunity against bacteria and Plasmodium. J Biol Chem 283, 3217-3223. 10.1074/jbc.M705873200.

Montell, C., and Zwiebel, L. (2016). Mosquito Sensory Systems. Advances in Insect Physiology. 10.1016/bs.aiip.2016.04.007.

Mulatier, M., Alou, L., Chander, F., Pennetier, C., Dormont, L., and Cohuet, A. (2018). Effect of DEET-multiple exposures on behavior and life history traits in the malaria mosquito Anopheles gambiae (s.s.). Parasite Vector 11, 432.

Nainu, F., Salim, E., Asri, R.M., Hori, A., and Kuraishi, T. (2019). Neurodegenerative disorders and sterile inflammation: lessons from a Drosophila model. J Biochem 166, 213-221. https://doi.org/10.1093/jb/mvz053. 
Nakhleh, J., Christophides, G.K., and Osta, M.A. (2017). The serine protease homolog CLIPA14 modulates the intensity of the immune response in the mosquito Anopheles gambiae. J Biol Chem 292, 18217-18226. 10.1074/jbc.M117.797787.

Nguyen, P.L., Vantaux, A., Hien, D.F., Dabire, K.R., Yameogo, B.K., Gouagna, L.C., Fontenille, D., Renaud, F., Simard, F., Constantini, C., et al. (2017). No evidence for manipulation of Anopheles gambiae, An. coluzzii and An. arabiensis host preference by Plasmodium falciparum. Sci Rep 25, 9415. doi: 10.1038/s41598-017-09821-x.

Ogola, E.O., Fillinger, U., Ondiba, I.M., Villinger, J., Masiga, D., Torto, B., and Tchouassi, D.P. (2018). Insights into malaria transmission among Anopheles funestus mosquitoes, Kenya. Parasite Vector 11, 577. https://doi.org/10.1186/s13071-018-3171-3.

Ohm, J.R., Teeple, J., Nelson, W.A., Thomas, M.B., Read, A.F., and Cator, L.J. (2016). Fitness consequences of altered feeding behavior in immune-challenged mosquitoes. Parasit Vectors 9, 113. https://doi.org/10.1186/s13071-016-1392-x.

Oliveira, G.d.O., Lieberman, J., and Barillas-Mury, C. (2012). Epithelial nitration by a peroxidase/NOX5 system mediates mosquito antiplasmodial immunity. Science 335, 856-859. 10.1126/science.1209678. 
Olliaro, P. (2008). Mortality associated with severe Plasmodium falciparum malaria increases with age. Clin Infect Dis 47, 158-160.

Oringanje, C., Delacruz, L.R., Han, Y., Luckhart, S., and Riehle, M.A. (2021). Overexpression of activated AMPK in Anopheles stephensi midgut impacts mosquito metabolism, reproduction and Plasmodium resistance. Genes 12, 119. 10.3390/genes12010119.

Osborne, R.H. (1996). Insect neurotransmission: neurotransmitters and their receptors. Pharmacol Ther 69, 117-142. 10.1016/0163-7258(95)02054-3.

Pattanakitsakul, S.-N., Boonnak, K., Auethavornanan, K., Jairungsri, A., Duangjinda, T., Puttatesk, P., Thongrungkiat, S., and Malasit, P. (2007). A new densovirus isolated from the mosquito Toxorhynchites splendens (Wiedemann) (Diptera: Culicidae). Southeast Asian J Trop Med Public Health 38, 283-293.

Pinheiro-Silva, R., Borges, L., Coelho, L.P., Cabezas-Cruz, A., Valdes, J.J., do Rosario, V., de la Fuente, J., and Domingos, A. (2015). Gene expression changes in the salivary glands of Anopheles coluzzii elicited by Plasmodium berghei infection. Parasite Vector 8, 485. https://doi.org/10.1186/s13071-015-1079-8.

Pinto, S.B., Kafatos, F.C., and Michel, K. (2007). The parasite invasion marker SRPN6 reduces sporozoite numbers in salivary glands of Anopheles gambiae. Cell Microbiol. Apr;10(4):891-8. doi: 10.1111/j.1462-5822.2007.01091.x. 
Pitts, R.J., Derryberry, S.L., Zhang, Z., and Zwiebel, L.J. (2017). Variant lonotropic Receptors in the Malaria Vector Mosquito Anopheles gambiae Tuned to Amines and Carboxylic Acids. Sci Rep 7, 40297. 10.1038/srep40297.

Pitts, R.J., Rinker, D., C, Jones, P.L., Rokas, A., and Zwiebel, L.J. (2011). Transcriptome Profiling of Chemosensory Appendages in the Malaria Vector Anopheles gambiae Reveals Tissue- and SexSpecific Signatures of Odor Coding. BMC Genomics 12. 10.1186/1471-2164-12-271.

Pumpuni, C.B., and Beier, J.C. (1995). Long-term survival of Plasmodium sporozoites in vitro. Parasitology Research 81, 178-180.

Ramirez, J.L., Muturi, E.J., Flor-Weiler, L.B., Vermillion, K., and Rooney, A.P. (2020). Peptidoglycan Recognition Proteins (PGRPs) Modulates Mosquito Resistance to Fungal Entomopathogens in a Fungal-Strain Specific Manner. Front Cell Infect Microbiol 9. doi: 10.3389/fcimb.2019.00465.

Rinker, D.C., Pitts, R.J., Zhou, X., Suh, E., Rokas, A., and Zwiebel, L.J. (2013a). Blood meal-induced changes to antennal transcriptome profiles reveal shifts in odor sensitivities in Anopheles gambiae. Proc Natl Acad Sci U S A 110, 8260-8265. 10.1073/pnas.1302562110. 
Rinker, D.C., Zhou, X., Pitts, R.J., Consortium, A.G.C., Rokas, A., and Zwiebel, L.J. (2013b).

Antennal transcriptome profiles of anopheline mosquitoes reveal human host olfactory specialization in Anopheles gambiae. BMC Genomics 14, 749. 10.1186/1471-2164-14-749.

Sandiford, S.L., Dong, Y., Pike, A., Blumberg, B., Bahia, A.C., and Dimopoulos, G. (2015).

Cytoplasmic actin is an extracellular insect immune factor which is secreted upon immune challenge and mediates phagocytosis and direct killing of bacteria, and is a Plasmodium Antagonist. PLoS

Pathog 11, e1004631. 10.1371/journal.ppat.1004631.

Schwarze, S.R., Weindruch, R., and Aiken, J.M. (1998). Oxidative stress and aging reduce COX I RNA and cytochrome oxidase activity in Drosophila. Free Radic Biol Med 25, 740-747. https://doi.org/10.1016/S0891-5849(98)00153-1.

Shuai, K., and Liu, B. (2003). Regulation of JAK-STAT signalling in the immune system. Nat Rev Immunol 3, 900-911. 10.1038/nri1226.

Sim, S., Ramirez, J.L., and Dimopoulos, G. (2012). Dengue Virus Infection of the Aedes aegypti Salivary Gland and Chemosensory Apparatus Induces Genes that Modulate Infection and BloodFeeding Behavior. PLoS Pathogens 8, e1002631. 10.1371/journal.ppat.1002631.t001. 
Sinka, M.E., Bangs, M.J., Manguin, S., Rubio-Palis, Y., Chareonviriyaphap, T., Coetzee, M., Mbogo, C.M., Hemingway, J., Patil, A.P., Temperley, W.H., et al. (2012). A global map of dominant malaria vectors. Parasite Vector 5. Artn 69 10.1186/1756-3305-5-69.

Smallegange, R.C., Qiu, Y.T., van Loon, J.J.A., and Takken, W. (2005). Synergism between ammonia, lactic acid and carboxylic acids as kairomones in the host-seeking behaviour of the malaria mosquito Anopheles gambiae sensu stricto (Diptera: Culicidae). Chem Senses 30, 145-152. 10.1093/chemse/bji010.

Smallegange, R.C., van Gemert, G.-J., van de Vegte-Bolmer, M., Gezan, S., Takken, W., Sauerwein, R.W., and Logan, J.G. (2013). Malaria Infected Mosquitoes Express Enhanced Attraction to Human Odor. PLoS ONE 8, e63602. 10.1371/journal.pone.0063602.g001.

Sokabe, T., and Tominaga, M. (2009). A temperature-sensitive TRP ion channel, Painless, functions as a noxious heat sensor in fruit flies. Commun Integr Biol 2, 170-173. 10.4161/cib.7708.

Sousa, G.L., Bishnoi, R., Baxter, R.H.G., and Povelones, M. (2020). The CLIP-domain serine protease CLIPC9 regulates melanization downstream of SPCLIP1, CLIPA8, and CLIPA28 in the malaria vector Anopheles gambiae. PLoS Pathog 16, e1008985. 10.1371/journal.ppat.1008985. 
Sparks, J.T., and Dickens, J.C. (2016). Electrophysiological Responses of Gustatory Receptor Neurons on the Labella of the Common Malaria Mosquito, Anopheles quadrimaculatus (Diptera: Culicidae). J Med Entomol. 10.1093/jme/tjw073.

Stanczyk, N.M., Brugman, V.A., Austin, V., Sanchez-Roman Teran, F., Gezan, S.A., Emery, M., Visser, T.M., Dessens, J.T., Stevens, W., Smallegange, R.C., et al. (2019). Species-specific alterations in Anopheles mosquito olfactory responses caused by Plasmodium infection. Scientific Reports 9, 3396.

Stanczyk, N.M., Mescher, M.C., and De Moraes, C.M. (2017). Effects of malaria infection on mosquito olfaction and behavior: extrapolating data to the field. Curr Opin Insect Sci 20, 7-12. 10.1016/j.cois.2017.02.002.

Swale, D.R., Sun, B., Tong, F., and Bloomquist, J.R. (2014). Neurotoxicity and mode of action of N, N-diethyl-meta-toluamide (DEET). PLoS ONE 9, e103713. 10.1371/journal.pone.0103713 PONE-D-13-55110 [pii].

Takken, W., and Verhulst, N.O. (2013). Host preferences of blood-feeding mosquitoes. Annu Rev Entomol 58, 433-453. 10.1146/annurev-ento-120811-153618. 
Thiery, I., Nicolas, L., Rippka, R., and Tandeau de Marsac, N. (1991). Selection of cyanobacteria isolated from mosquito breeding sites as a potential food source for mosquito larvae. Appl Environ Microbiol 57, 1354-1359.

Tower, J. (2010). Heat shock proteins and Drosophila aging. 46, 355-362.

10.1016/j.exger.2010.09.002.

Trpis, M., McClelland, G.A., Gillett, J.D., Teesdale, C., and Rao, T.R. (1973). Diel periodicity in the landing of Aedes aegypti on man. Bull World Health Organ 48, 623-629.

Ulgherait, M., Rana, A., Rera, M., Graniel, J., and Walker, D.W. (2014). AMPK modulates tissue and organismal aging in a non-cell-autonomous manner. Cell Rep 8, 1767-1789.

10.1016/j.celrep.2014.08.006.

Untergasser, A., Cutcutache, I., Koressaar, T., Ye, J., Faircloth, B.F., Remm, M., and Rozen, S.G. (2012). Primer3--new capabilities and interfaces. Nucleic Acids Res 40, e115. 10.1093/nar/gks596.

Vantaux, A., de Sales Hien, D.F., Yameogo, B., Dabire, K.R., Thomas, F., Cohuet, A., and Lefevre, T. (2015). Host-seeking behaviors of mosquitoes experimentally infected with sympatric field isolates of the human malaria parasite Plasmodium falciparum: no evidence for host manipulation. Front Ecol Evol. https://doi.org/10.3389/fevo.2015.00086. 
Vaughan, J.A., Noden, B.H., and Beier, J.C. (1994). Sporogonic development of cultured Plasmodium falciparum in six species of laboratory-reared Anopheles mosquitotes. Am. J. Trop. Med. Hyg. 51, 233-243.

Verhulst, N.O., Mbadi, P.A., Bukovinszkiné Kiss, G., Mukabana, W.R., van Loon, J.J.A., Takken, W., and Smallegange, R.C. (2011). Improvement of a synthetic lure for Anopheles gambiae using compounds produced by human skin microbiota. Malaria Journal 10, 28-36.

Vogt, R.G., Sparks, J.T., Fandino, R.A., and Ashourian, K.T. (2021). Reflections on antennal proteins: The evolution of pheromone binding proteins; diversity of pheromone degrading enzymes; and the distribution and behavioral roles of SNMPs. Insect Pheromone Biochemisty and Molecular Biology, 675-707. https://doi.org/10.1016/B978-0-12-819628-1.00021-3.

Vrba, J., Trtkova, K., and Ulrichova, J. (2011). HDAC inhibitors sodium butyrate and sodium valproate do not affect human ncor1 and ncor2 gene expression in HL-60 cells. Biomed Pap Med Fac Univ Palacky Olomouc Czech Repub 155, 259-262. 10.5507/bp.2011.033.

Wang, T., Si, F., He, Z., and Chen, B. (2018). Genome-wide identification, characterization and classification of ionotropic glutamate receptor genes (iGluRs) in the malaria vector Anopheles sinensis (Diptera: Culicidae). Parasit Vectors 11, 24. 
Wang, X., Hou, Y., Saha, T.T., Pei, G., Raikhel, A.S., and Zou, Z. (2017). Hormone and receptor interplay in the regulation of mosquito lipid metabolism. Proc Natl Acad Sci U S A. 114, E2709-2718. https://doi.org/10.1073/pnas.1619326114.

Waterhouse, R.M., Povelones, M., and Christophides, G.K. (2010). Sequence-structure-function relations of the mosquito leucine-rich repeat immune proteins. BMC genomics 11,531 . 10.1186/1471-2164-11-531.

WHO (2020). World Malaria Report 2020: 20 Years of global progress and challenges.

Wolff, G.H., and Riffell, J.A. (2018). Olfaction, experience and neural mechanisms underlying mosquito host preference. J Exp Biol 221, jeb157131. https://doi.org/10.1242/jeb.157131.

Wullschleger, S., Loewith, R., and Hall, M.N. (2006). TOR signaling in growth and metabolism. Cell 124, 471-484. S0092-8674(06)00108-5 [pii] 10.1016/j.cell.2006.01.016.

Yan, Y., and Hillyer, J.F. (2019). Complement-like proteins TEP1, TEP3 and TEP4 are positive regulators of periostial hemocyte aggregation in the mosquito Anopheles gambiae. Insect Biochem Mol Biol 107, 1-9. doi: 10.1016/j.ibmb.2019.01.007. 
A Control Bloodmeal

(non-infected)

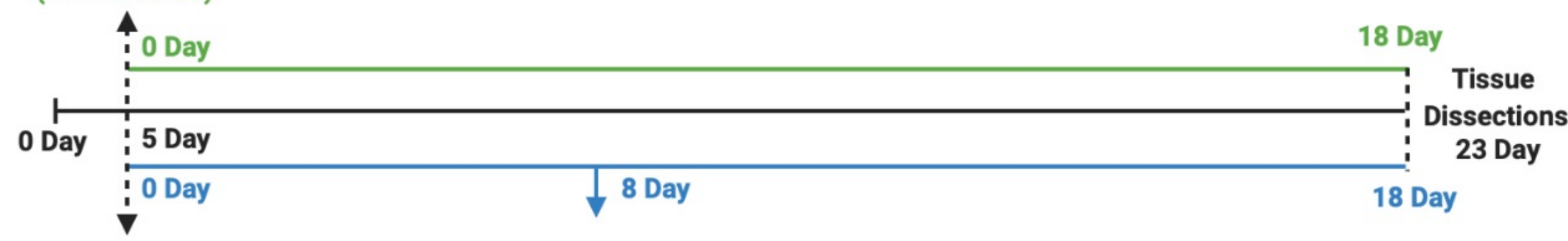

Treatment Bloodmeal

Midgut Oocyst Counts

Sporogonic Cycle Complete

( $P f$ infected)

Mean No. $=1.3$

Infectious-Stage

B
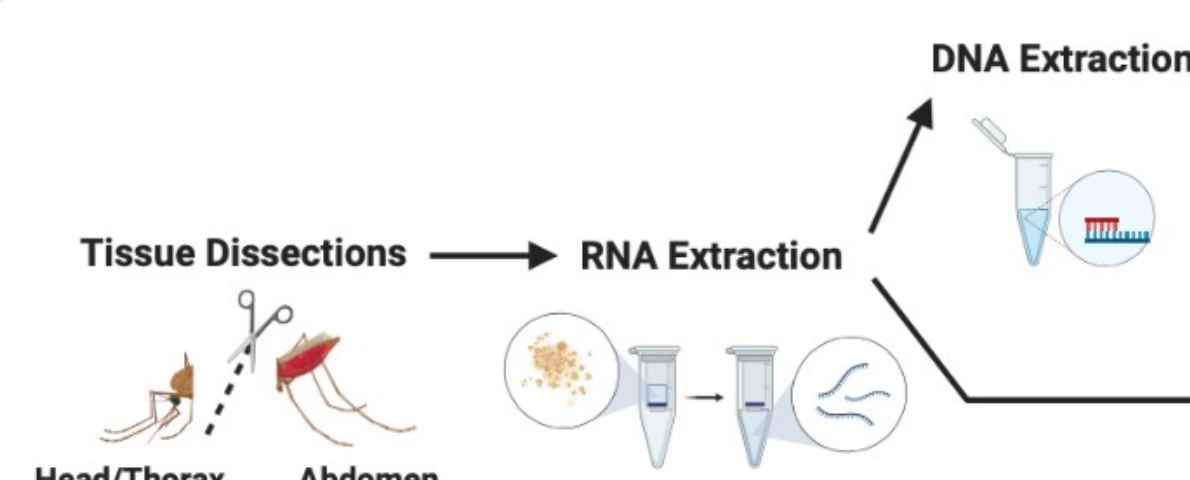

Head/Thorax

Abdomen

Treatment Protocol

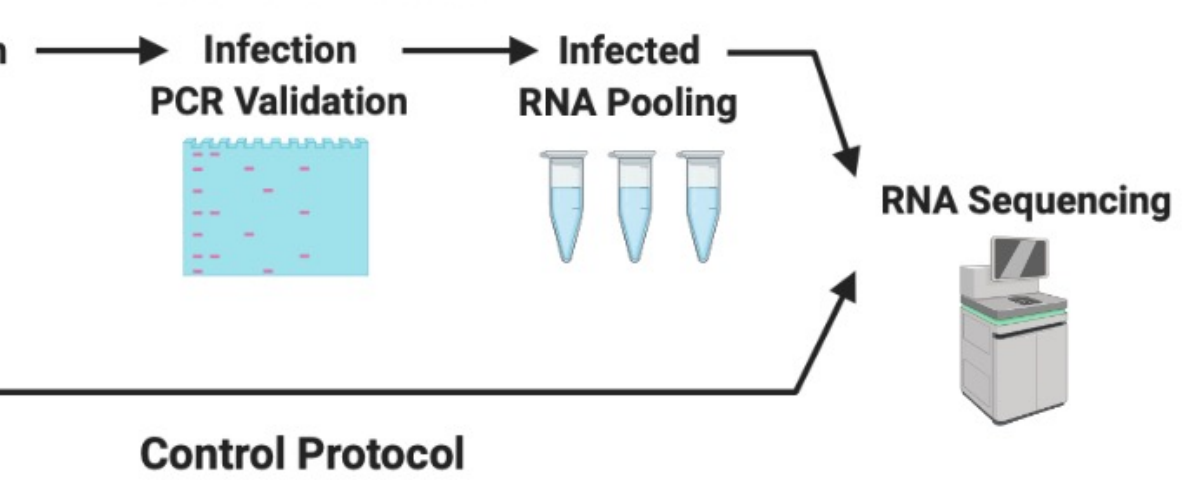

Figure 1 


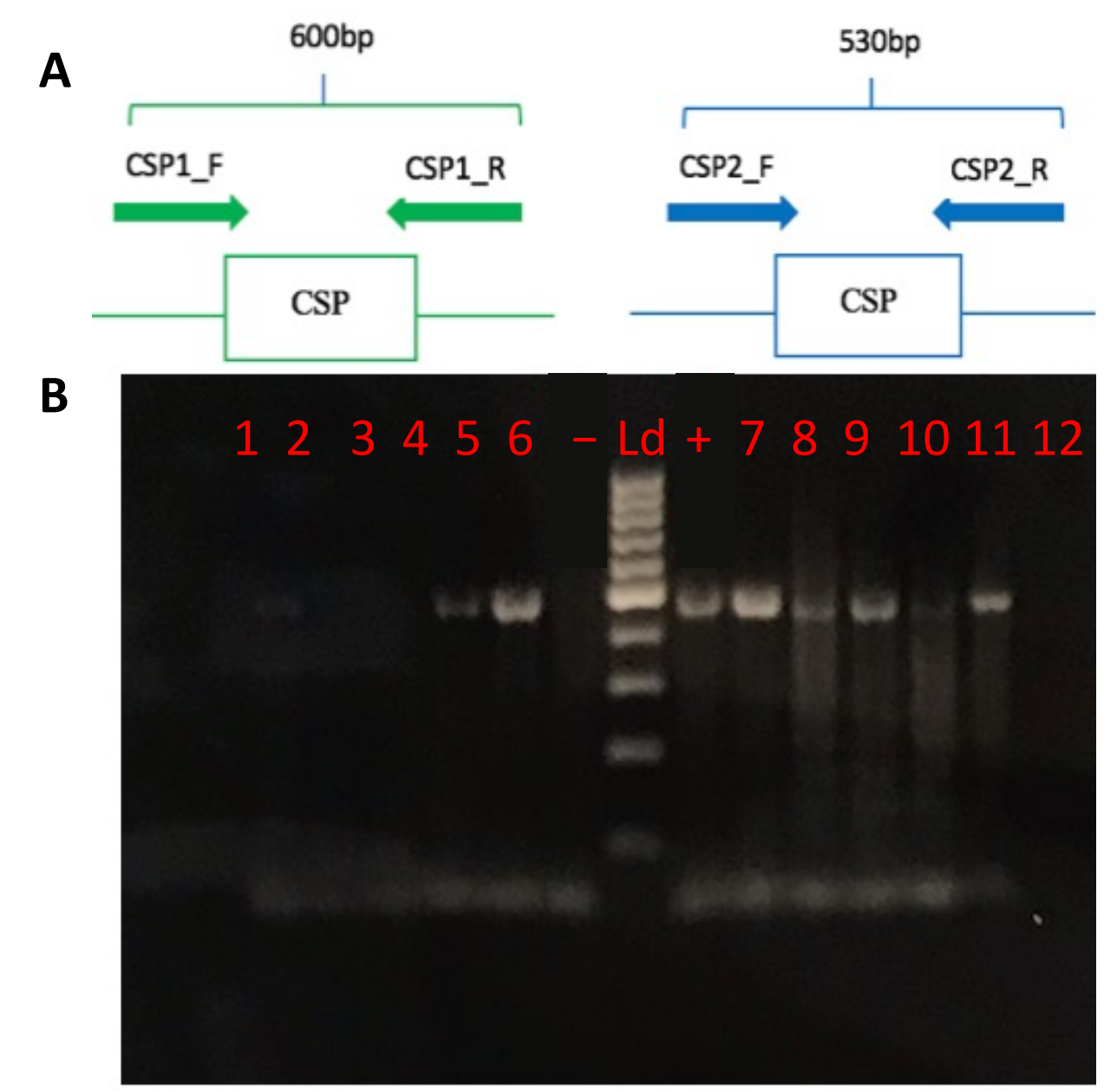

Figure 2 
A

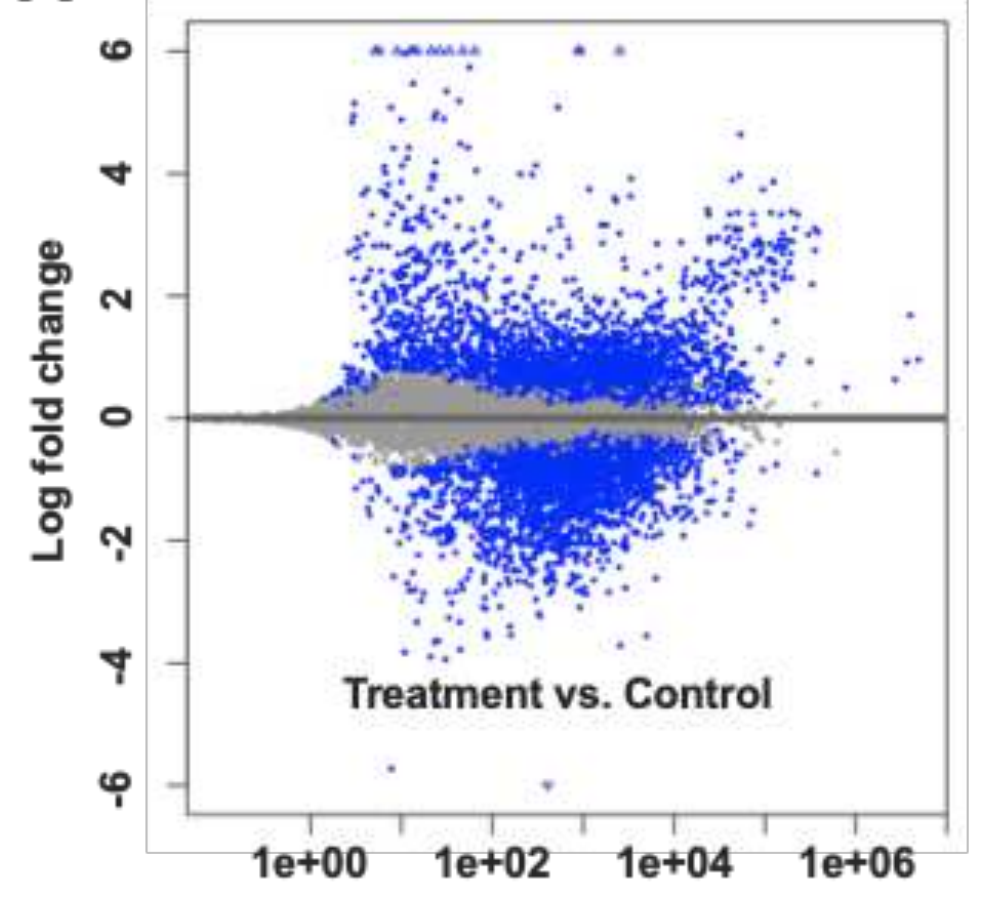

Mean of normalized counts
B

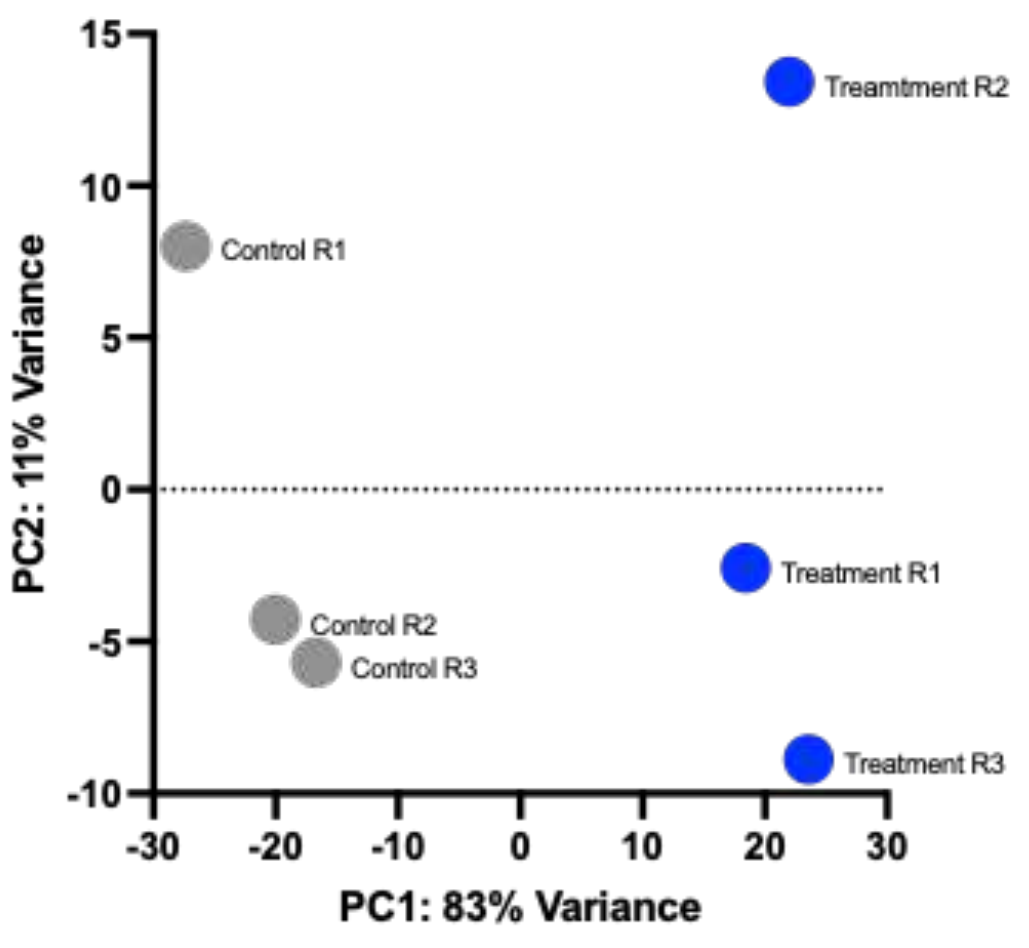

\section{Figure 3}


A

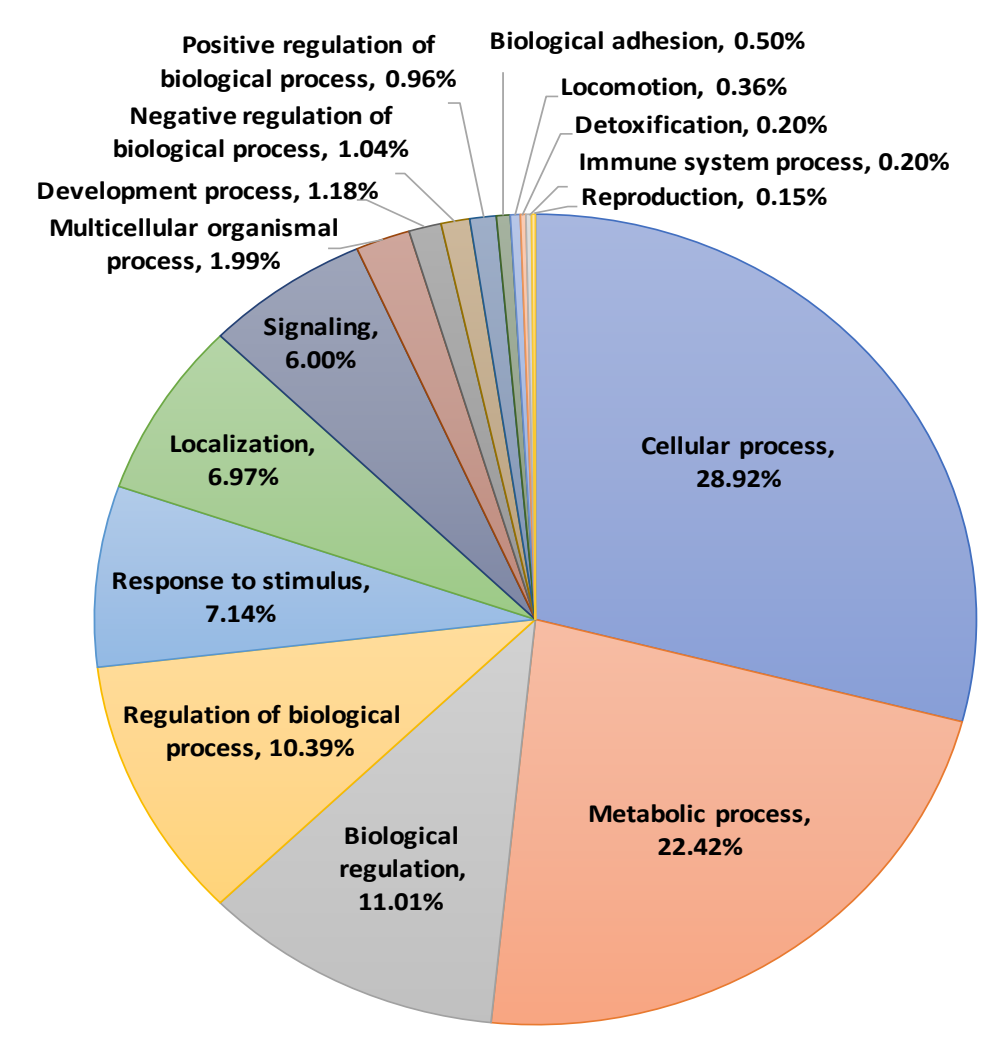

B
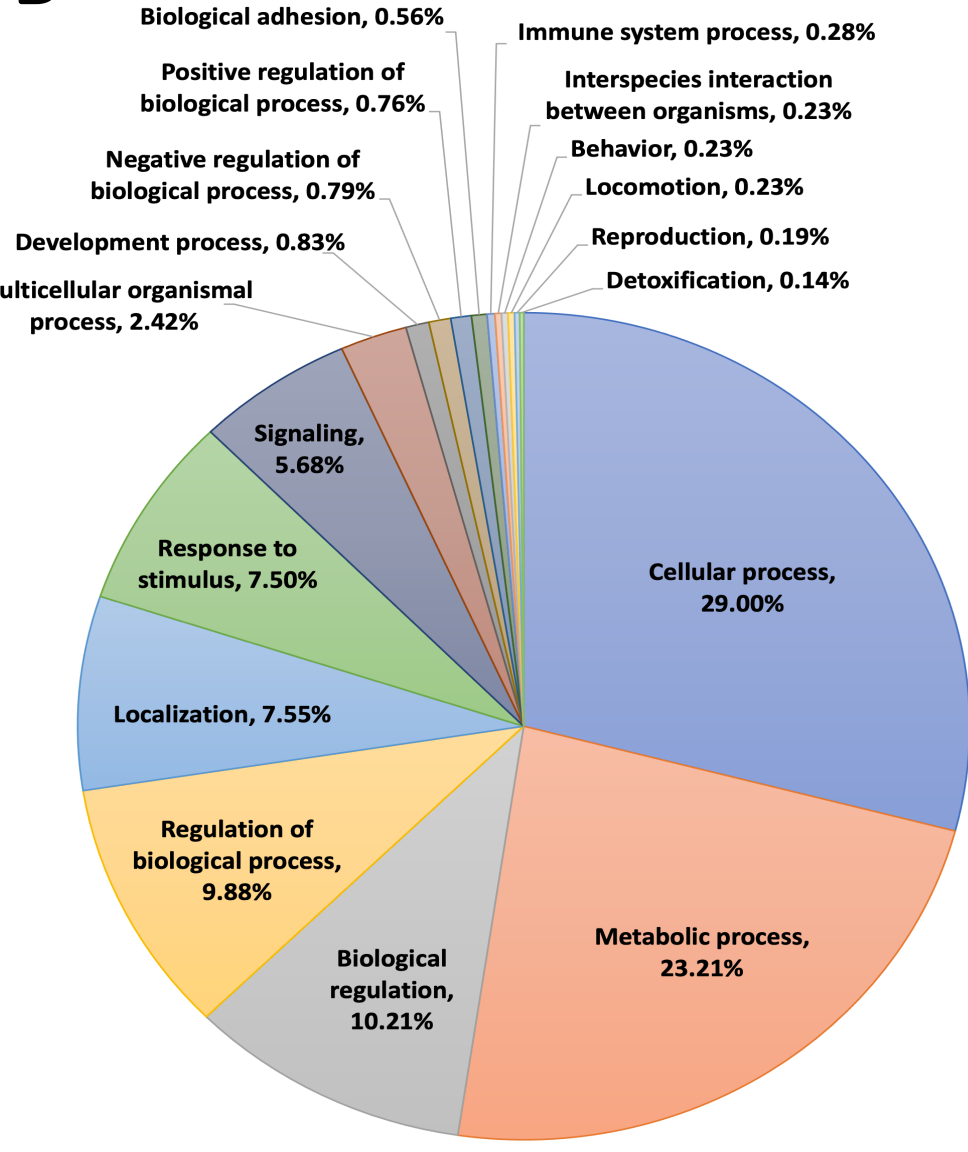

C

Biological adhesion, $0.81 \%$

\begin{tabular}{l|l} 
Positive regulation of biological process,... & Locomotion, $0.47 \%$
\end{tabular}

Negative regulation of biological process, $1.23 \%$ Detoxification, $0.25 \%$

Developmental process, $1.44 \%$

Growth, $0.21 \%$

Reproduction, $0.21 \%$

Signaling, $6.23 \%$

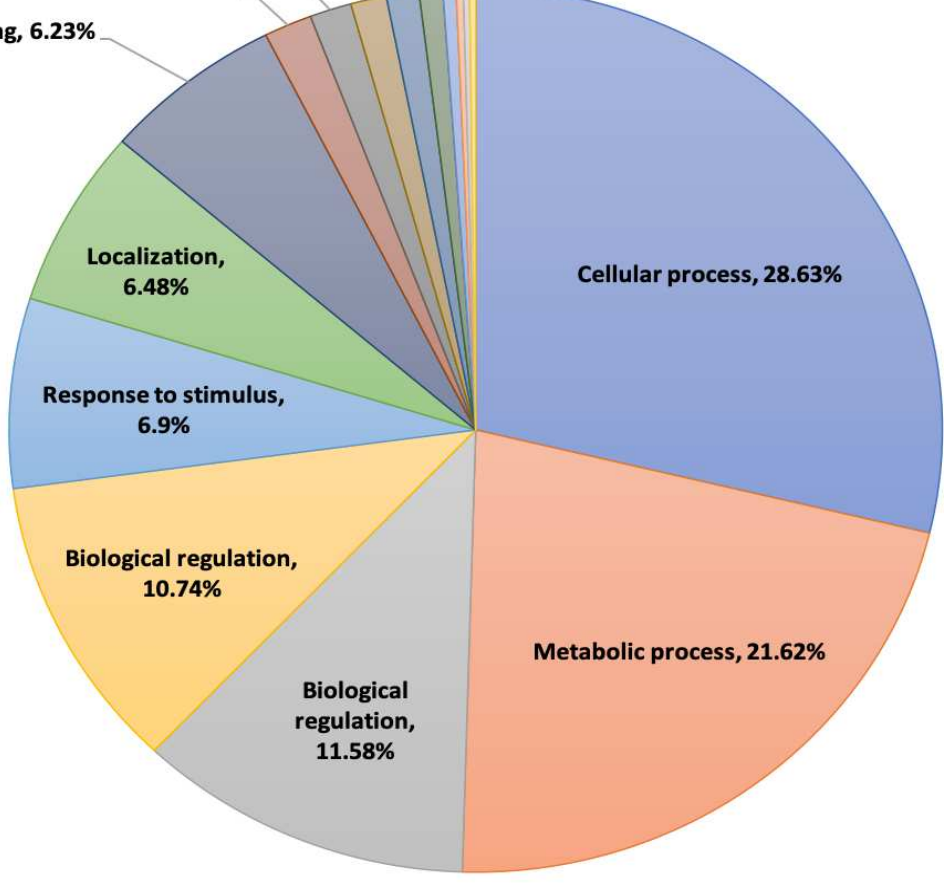

\section{Figure 4}




\begin{tabular}{|l} 
AgSAP2- \\
AgOBP13- \\
AgCSP4- \\
AgOBP26- \\
AgOBP29- \\
AgOBP28- \\
AgOBP54- \\
AgOBP21- \\
AgOBP71- \\
AgOBP46- \\
AgOBP11- \\
AgOBP23- \\
AgOBP33- \\
AgOBP51- \\
AgOBP67- \\
AgOBP8- \\
AgOBP38- \\
AgOBP37- \\
AgOBP34- \\
AgOBP68- \\
AgOBP68- \\
AgOBP43- \\
AgOBP35- \\
AgOBP12- \\
AgOBP49- \\
AgOBP36- \\
AgOBP42- \\
AgOBP52-
\end{tabular}
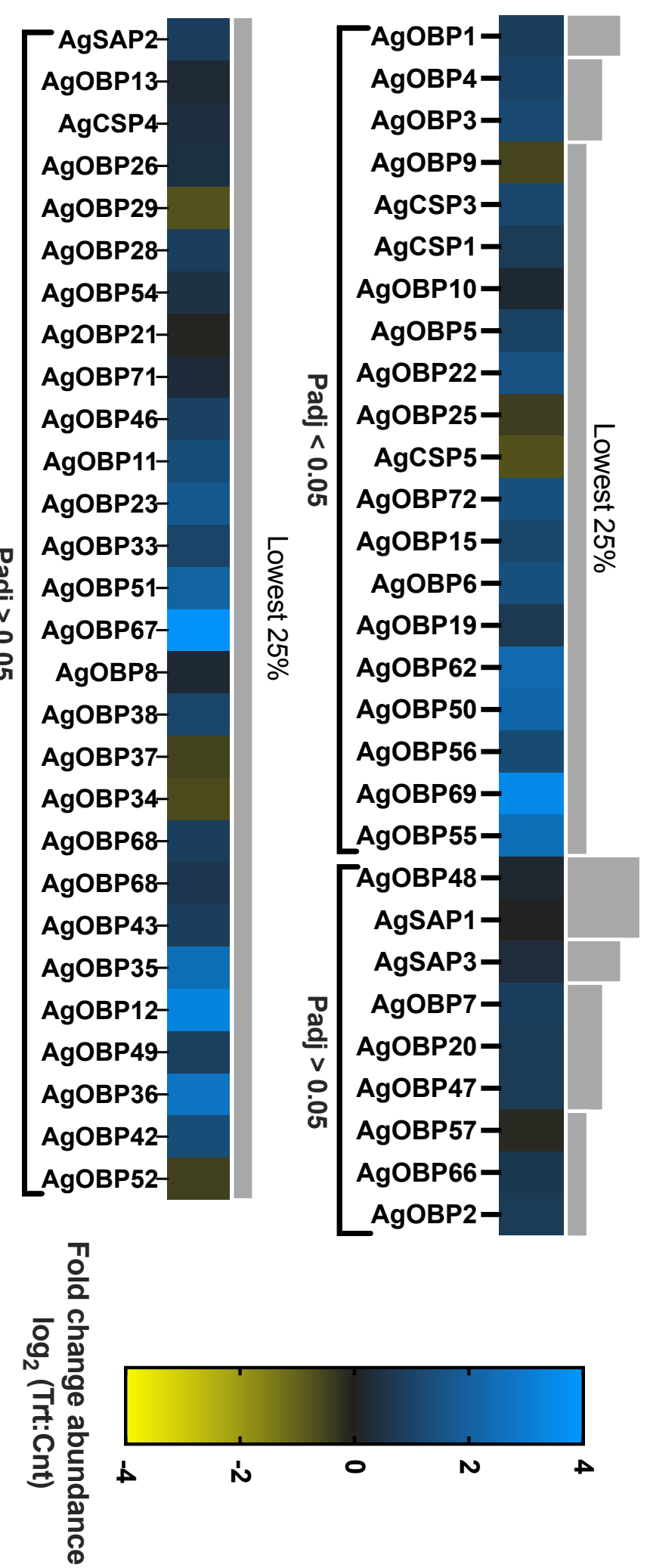

\begin{tabular}{|c|} 
AgOR57- \\
AgOR1- \\
AgOR36- \\
AgOR2- \\
AgOR59- \\
AgOR66- \\
AgOR69- \\
AgOR63- \\
AgOR31- \\
AgOR60- \\
AgOR48- \\
AgOR16- \\
AgOR62- \\
AgOR22- \\
AgOR49- \\
AgOR33- \\
AgOR6- \\
AgOR27- \\
AgOR82A- \\
AgOR41- \\
AgOR47- \\
AgOR72- \\
AgOR14- \\
AgOR26- \\
AgOR64- \\
AgOR18- \\
AgOR77- \\
AgOR4- \\
AgOR37- \\
AgOR58- \\
AgOR45- \\
AgOR65- \\
AgOR30- \\
AgOR46- \\
AgOR71- \\
\hline
\end{tabular}

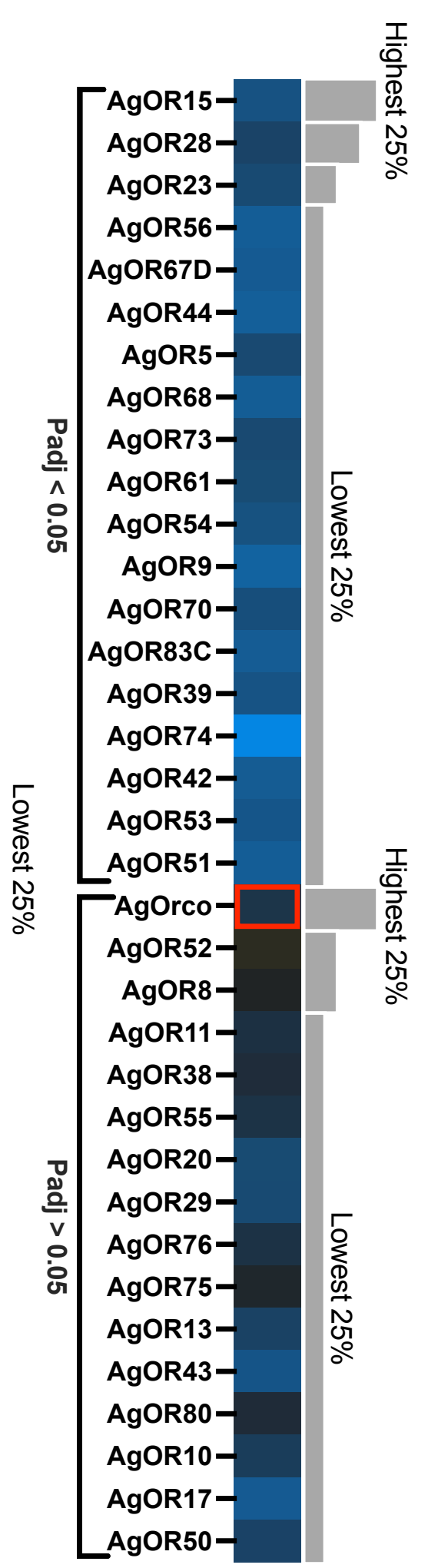


geranyl-acetone
benzaldehyde

$$
\text { E2-hexenal- }
$$

6-methyl-5-hepten-2-one-

ammonia$$
\text { 1-butanol- }
$$

(-)-fenchone -

2-butanone-

ethyl-hexanoate-

soamyl-acetate

4-ethylphenol-

benzyl-acetate

(-)-carvone-

amyl-acetate

achocete-

hexanal-

citronellal

3-octanone-

methyl-octanoate-

heptanal-

methyl-benzoate

nonanat-

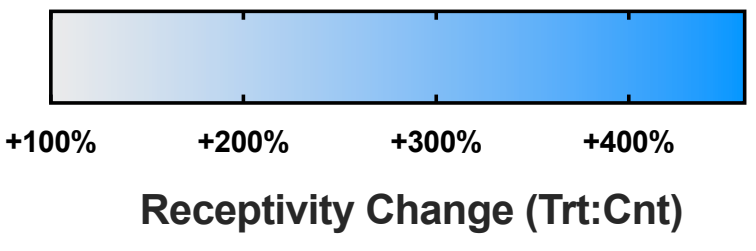

Figure 6 


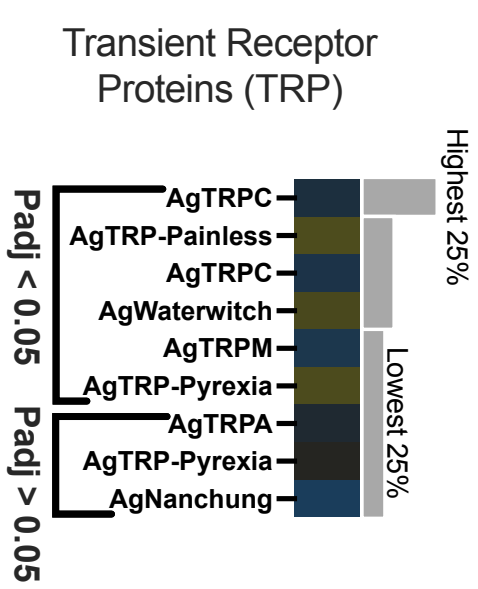

Pickpocket

Proteins (PPK)

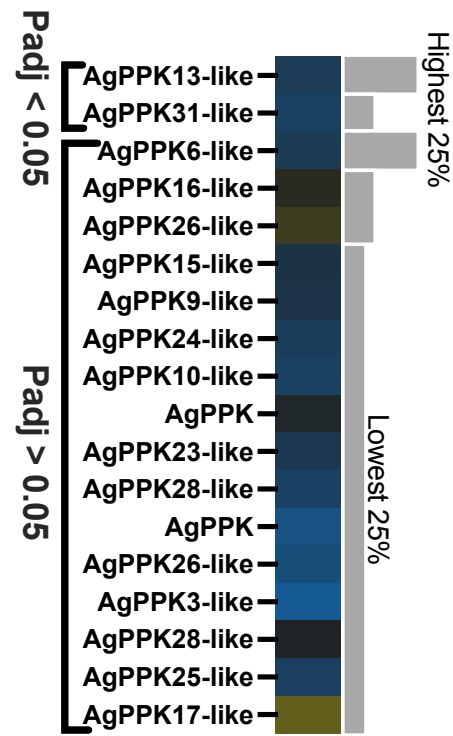

\section{끙}

or

을 ํำ

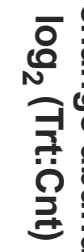
N 0 음 .

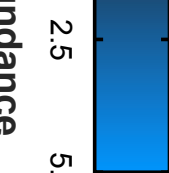
0 폴

○

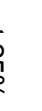
( 
Acetylcholine Receptors

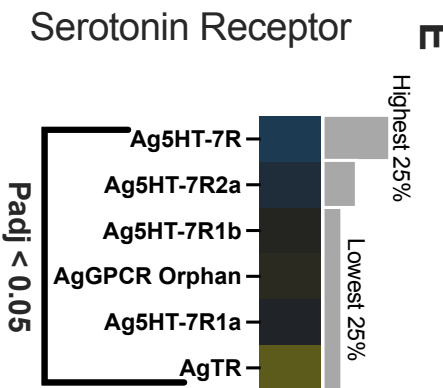

Octopamine Receptor T

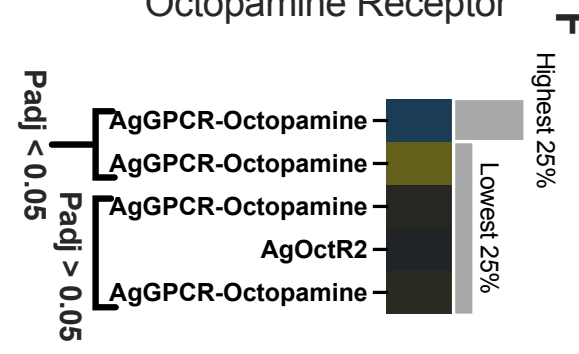

Dopamine Receptor

ค
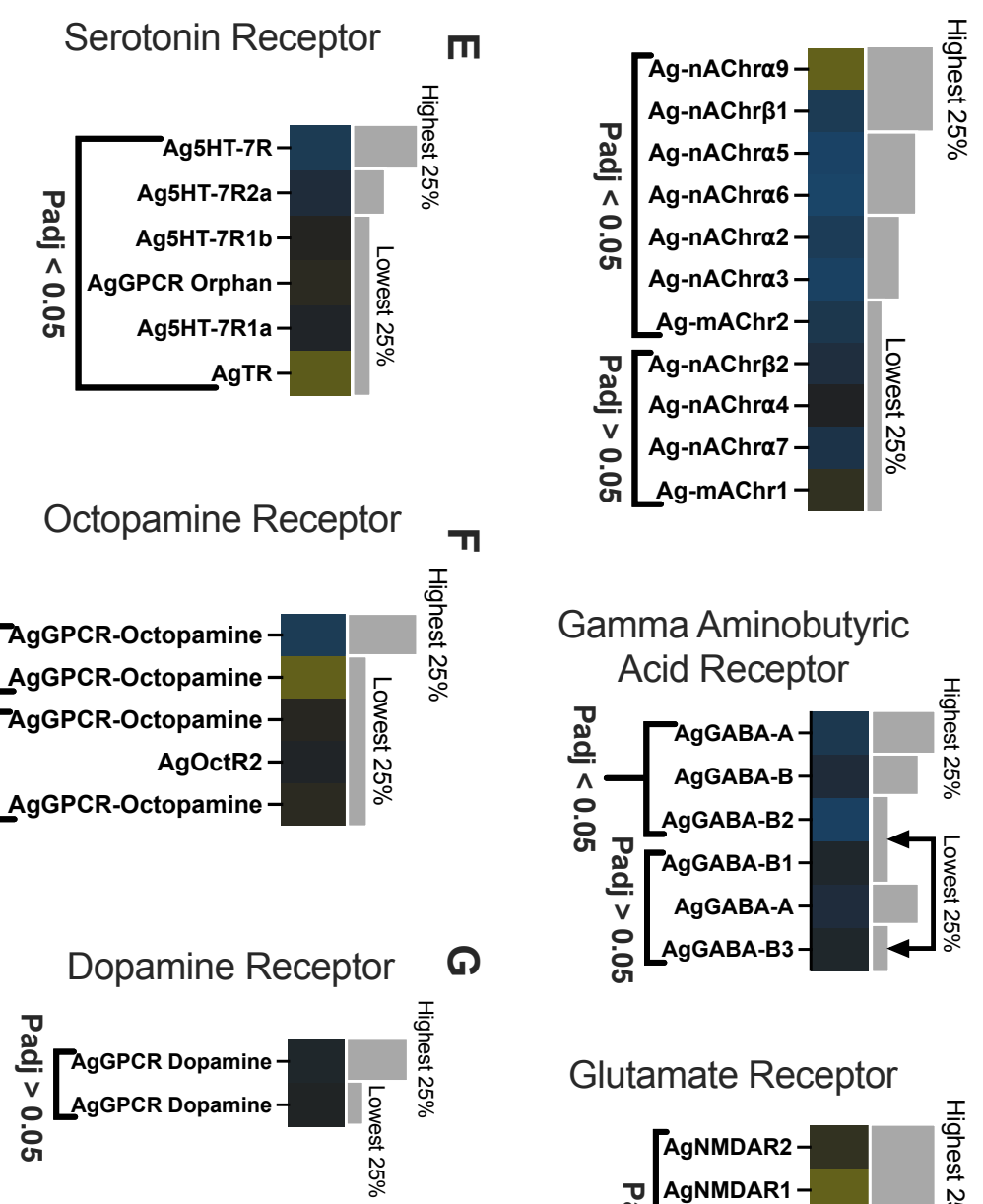

Gamma Aminobutyric Acid Receptor
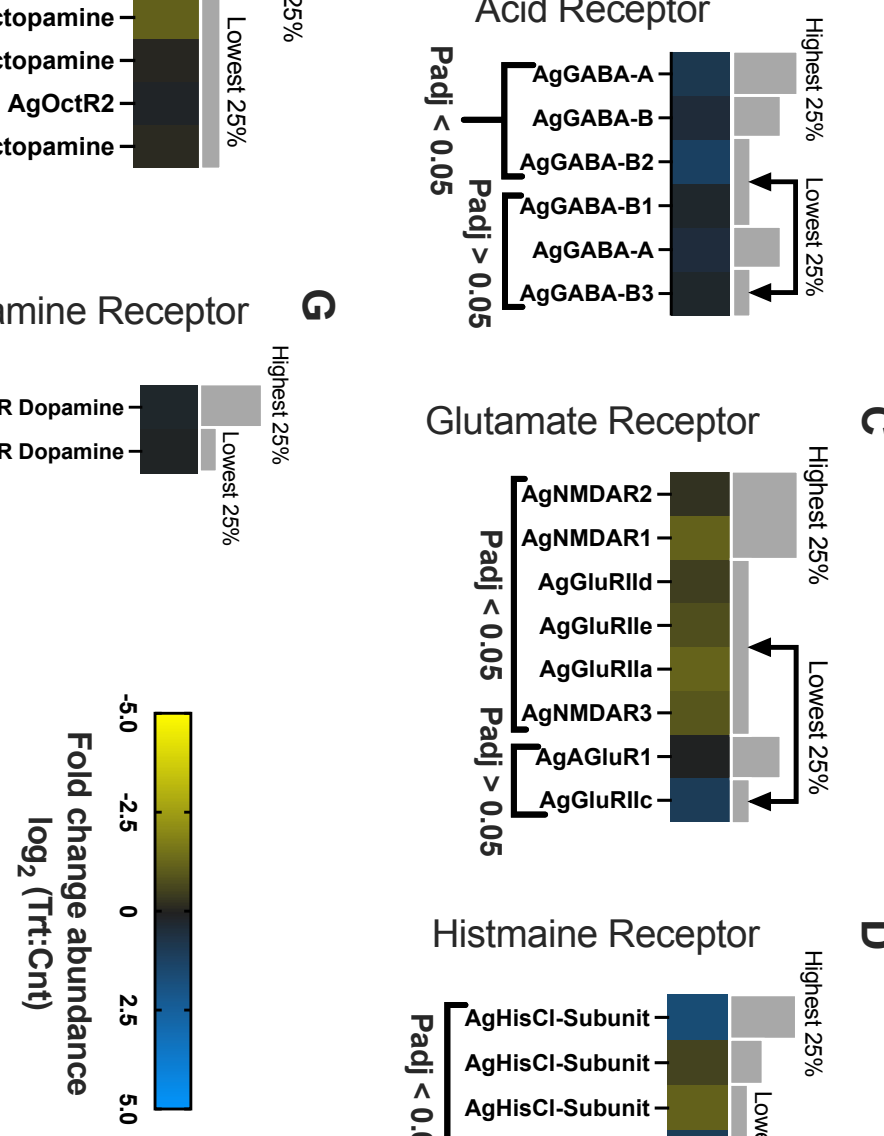

Histmaine Receptor

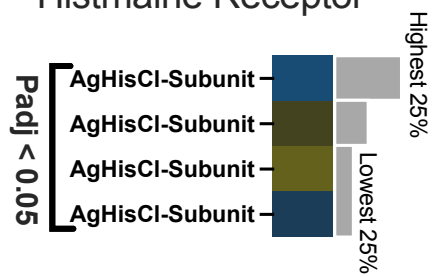




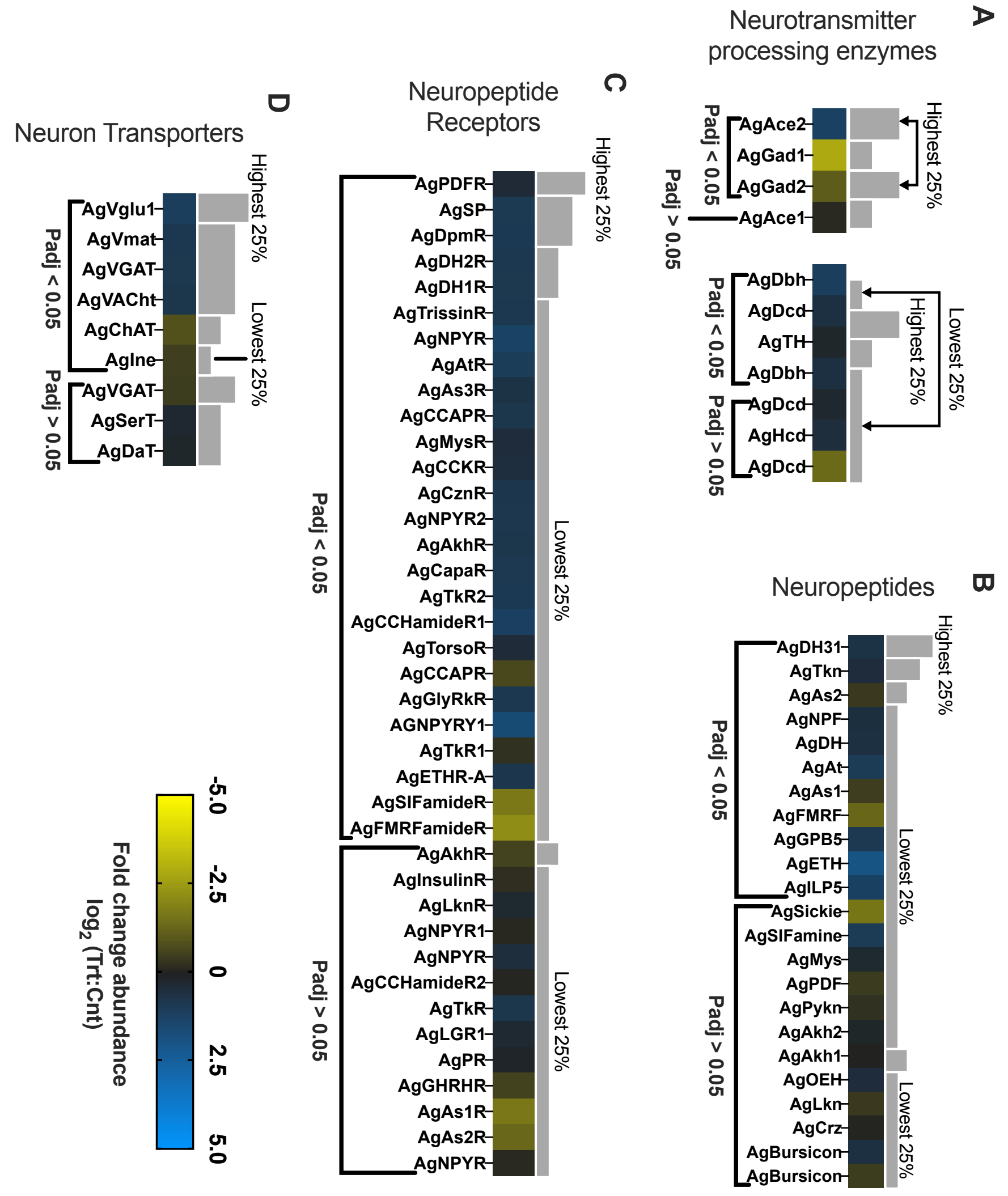


$\frac{T}{6}$
$\frac{\bar{C}}{\bar{D}}$
$\vec{\square}$

IMD \& JAK-STAT $\quad \boldsymbol{\omega}$

Toll \& Spaetzle (SPZ) D
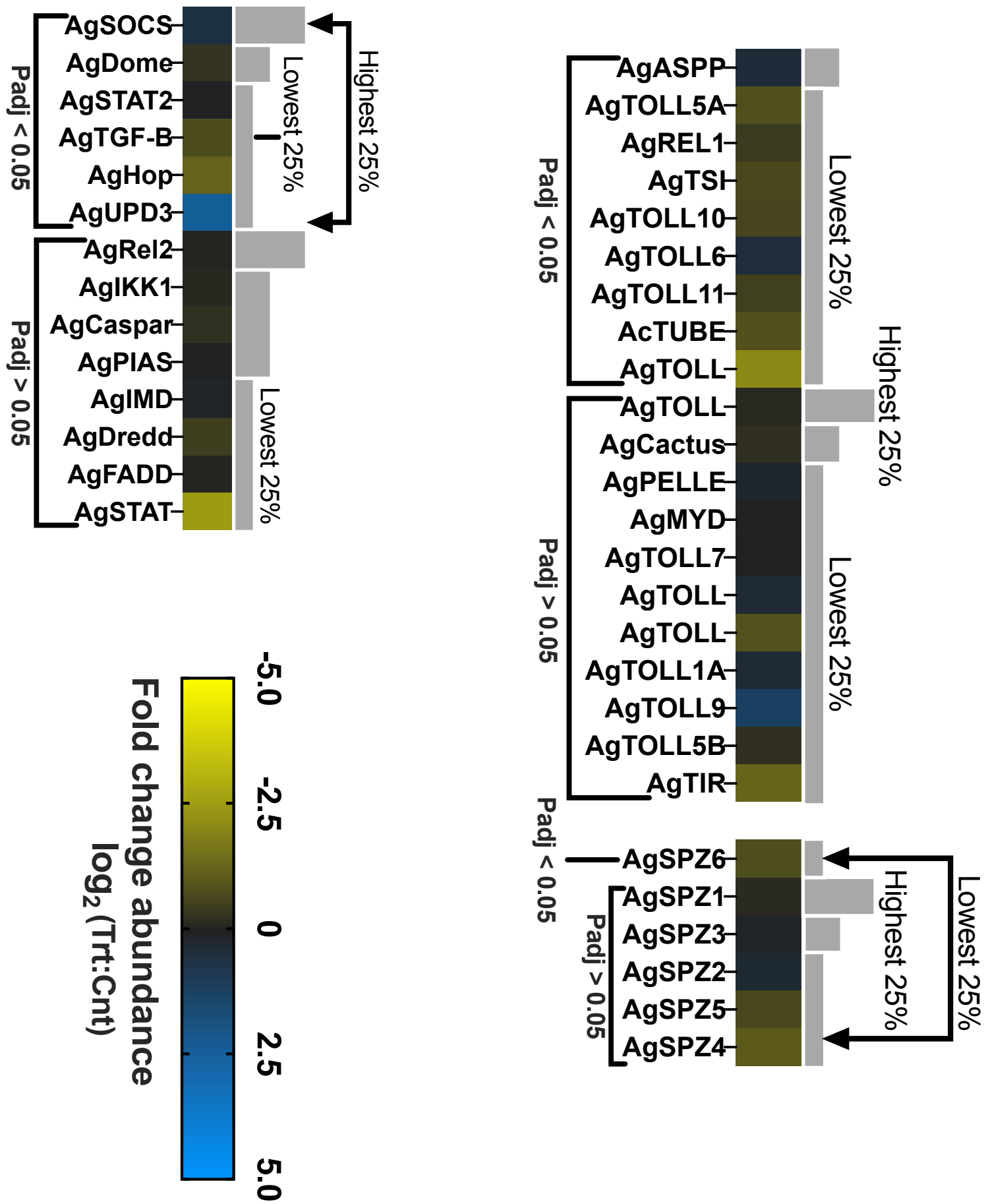

or 


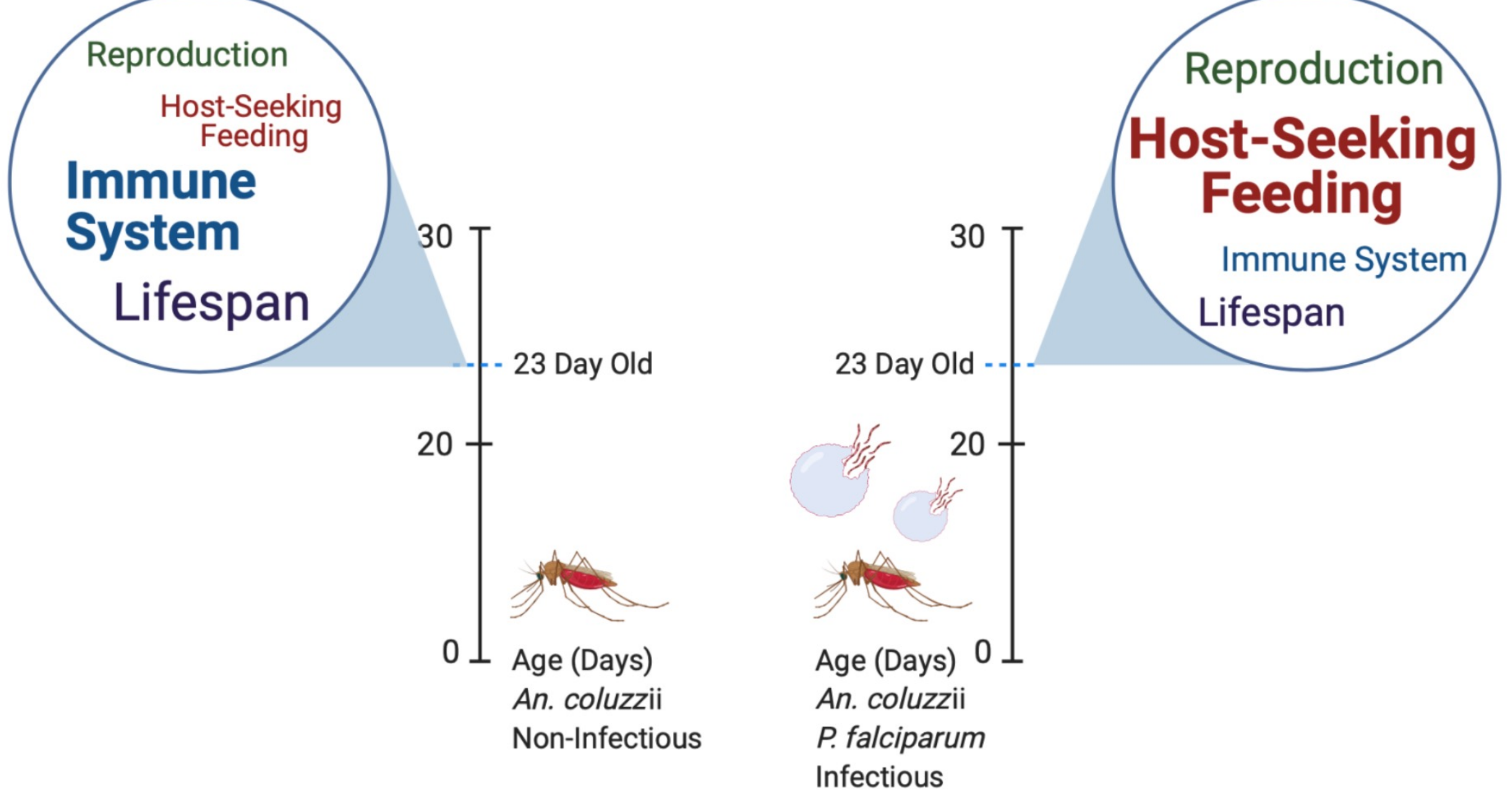




\begin{tabular}{|c|c|c|c|c|c|c|c|}
\hline \multicolumn{2}{|c|}{ An. gambiae s.s Lab Lines } & 1 & 2 & 3 & 4 & 5 & 6 \\
\hline \multirow{5}{*}{$\begin{array}{c}\text { P. falciparum } \\
0.15 \%\end{array}$} & Median & 29 & 12.5 & 22 & 11.5 & 13 & 13 \\
\hline & Mean & 25.7 & 14.3 & 32.6 & 13 & 12.9 & 11.3 \\
\hline & Range & 45 & 45 & 53 & 25 & 19 & 22 \\
\hline & $N=$ & 7 & 10 & 7 & 8 & 7 & 7 \\
\hline & Prevalence & $100 \%$ & $90 \%$ & $100 \%$ & $100 \%$ & $85.7 \%$ & $85.7 \%$ \\
\hline \multirow{5}{*}{$\begin{array}{c}\text { P. falciparum } \\
0.01 \%\end{array}$} & Median & 4.5 & 3 & 2.5 & 4 & \multirow{5}{*}{ N/A } & \multirow{5}{*}{ N/A } \\
\hline & Mean & 4.8 & 4.4 & 2.8 & 5.2 & & \\
\hline & Range & 10 & 11 & 1 & 1 & & \\
\hline & $N=$ & 8 & 7 & 8 & 9 & & \\
\hline & Prevalence & $87.5 \%$ & $85.7 \%$ & $87.5 \%$ & $88.9 \%$ & & \\
\hline \multirow{5}{*}{$\begin{array}{c}\text { P. falciparum } \\
0.008 \%\end{array}$} & Median & 1 & 1 & 1 & 1.1 & 1 & 1 \\
\hline & Mean & 1 & 1.7 & 1.2 & 1.4 & 1.1 & 1.4 \\
\hline & Range & 3 & 6 & 5 & 3 & 3 & 4 \\
\hline & $\mathrm{N}=$ & 7 & 9 & 9 & 11 & 10 & 12 \\
\hline & Prevalence & $57.1 \%$ & $66.7 \%$ & $55.6 \%$ & $63.6 \%$ & $60.0 \%$ & $66.7 \%$ \\
\hline
\end{tabular}

\section{Table 1}




\begin{tabular}{|l|c|c|c|c|c|c|}
\hline Exp. Line & Replicate & Total No. Reads & Mapped Reads & No. Transcripts & PCC (r) & Coverage \\
\hline \multirow{3}{*}{ Treatment } & $\mathrm{T} 1$ & $65,613,838$ & $54,459,486(83 \%)$ & $12,194(77 \%)$ & 0.9429 & $70 \mathrm{x}$ \\
\cline { 2 - 5 } & $\mathrm{T} 2$ & $123,249,032$ & $102,296,697(83 \%)$ & $12,799(81 \%)$ & & $131 \mathrm{x}$ \\
\cline { 2 - 5 } & $\mathrm{T} 3$ & $65,668,388$ & $55,818,130(85 \%)$ & $12,533(79 \%)$ & & $70 \mathrm{x}$ \\
\cline { 3 - 5 } Control & $\mathrm{C} 1$ & 166,457374 & $123,178,457(74 \%)$ & $12,848(81 \%)$ & 0.9770 & $177 \mathrm{x}$ \\
\cline { 2 - 5 } & $\mathrm{C} 2$ & $110,612,110$ & $81,852,961(74 \%)$ & $12,625(80 \%)$ & & $118 \mathrm{x}$ \\
\cline { 2 - 5 } & $\mathrm{C} 3$ & $149,580,984$ & $107,698,308(72 \%)$ & $12,719(81 \%)$ & & $160 \mathrm{x}$ \\
\hline
\end{tabular}




\section{Supplementary Files}

This is a list of supplementary files associated with this preprint. Click to download.

- CarretalNSciRepFigureS1.pdf

- CarretalSupInfo4NSciRep.xlsx 